\title{
Nonlinear 3D cosmic web simulation with heavy-tailed generative adversarial networks
}

\author{
Richard M. Feder®* \\ California Institute of Technology, Division of Physics, Math, and Astronomy, \\ 1200 East California Boulevard, Pasadena, California 91125, USA \\ Philippe Berger@ \\ Jet Propulsion Laboratory, California Institute of Technology, Pasadena, California 91109, USA \\ George Stein \\ Berkeley Center for Cosmological Physics, University of California, Berkeley, California 94720, USA
}

(Received 24 May 2020; accepted 23 September 2020; published 4 November 2020)

\begin{abstract}
Fast and accurate simulations of the nonlinear evolution of the cosmic density field are a major component of many cosmological analyses, but the computational time and storage required to run them can be exceedingly large. For this reason, we use generative adversarial networks (GANs) to learn a compressed representation of the 3D matter density field that is fast and easy to sample, and for the first time show that GANs are capable of generating samples at the level of accuracy of other conventional methods. Using subvolumes from a suite of GADGET-2 $N$-body simulations, we demonstrate that a deepconvolutional GAN can generate samples that capture both large- and small-scale features of the matter density field, as validated through a variety of $n$-point statistics. The use of a data scaling that preserves high-density features and a heavy-tailed latent space prior allow us to obtain state of the art results for fast 3D cosmic web generation. In particular, the mean power spectra from generated samples agree to within $5 \%$ up to $k=3$ and within $10 \%$ for $k \leq 5$ when compared with $N$-body simulations, and similar accuracy is obtained for a variety of bispectra. By modeling the latent space with a heavy-tailed prior rather than a standard Gaussian, we better capture sample variance in the high-density voxel PDF and reduce errors in power spectrum and bispectrum covariance on all scales. Furthermore, we show that a conditional GAN can smoothly interpolate between samples conditioned on redshift. Deep generative models, such as the ones described in this work, provide great promise as fast, low-memory, high-fidelity forward models of large-scale structure.
\end{abstract}

DOI: 10.1103/PhysRevD.102.103504

\section{INTRODUCTION}

Modeling the evolution of the large-scale structure of the Universe is a crucial part of many cosmological analyses. The initial linear growth of structures becomes highly nonlinear at later times and smaller scales, such that analytical approaches are unable to accurately predict observations in those regimes. For this reason, numerical simulations [1] of a representative volume of the universe are required to bridge the gap between the early universe and present day observations.

$N$-body simulations, which numerically solve the Poisson equation, are a standard operating procedure for modeling large-scale structure. These methods are exact in the sense that they directly simulate the gravitational evolution of some set of collisionless dark matter

rfederst@caltech.edu "particles," but the required mass and time resolution to make model predictions at scale is computationally expensive and requires significant data storage. Next-generation cosmological inferences will require thousands of simulations on the largest scales in order to constrain models like $\Lambda \mathrm{CDM}$ at the subpercent level [2], a task that is currently intractable to all but the largest future computational grants when using standard $N$-body methods. Another consideration is memory storage. At the moment, storing thousands of full scale $N$-body simulation snapshots is only feasible for petabyte-scale data storage facilities. Even with access to such resources, the analysis workflow may be cumbersome if one has to repeatedly transfer simulations between a cluster and local machine.

The need for fast and accurate simulations at a cheaper computational cost has led to the development of many "approximate" methods of cosmic structure simulation [3-11], which implement various physical approximations 
of gravity to decrease the simulation run-time and/or memory requirement while attempting to maintain a high degree of accuracy. These methods have shown to be very useful, but they still require a non-negligible amount of computation time, and the accuracy of their results has room to be improved upon.

One may then wonder if moving past physical approximations of gravity, and instead allowing for arbitrary nonlinear mappings from initial conditions (or some other prior distribution) to present day observables, could provide more accurate simulations at a smaller computational cost. This application of machine learning techniques to aid in the efficient modeling of large-scale structure has not gone uninvestigated, and there has recently been great interest in the use of machine learning across the field ${ }^{1}$ [12]. Image-to-image mapping techniques that transform initial conditions to the final structures [13-15] or augment dark matter-only simulation results with various physical effects not present in the simulation [16-21] have shown promise. However, these still require the generation of initial conditions, or the full simulation output, respectively. A fully generative model that does not require these intermediate steps is desirable.

In particular, generative adversarial networks (GANs) have garnered attention in recent years due to their ability to generate fast realizations of large-scale structure. GANs are one class of deep generative modeling techniques that seeks to learn a probability distribution over an arbitrary data distribution, doing so through a two-player adversarial optimization scheme [22] (see Sec. II for more details).

To date, there have been a number of successful implementations of cosmological modeling tasks using GANs. They have been used to generate accurate weak lensing convergence maps as evaluated through the power spectrum and a variety of Minkowski functionals [23], to model 2D slices of the cosmic density field from 3D $\mathrm{N}$-body simulations [24], and to generate 3D realizations of neutral hydrogen [25]. These methods learn a compressed representation of the data distribution and sample from the corresponding lower-dimensional latent space to produce new samples.

There are also a number of in-painting and superresolution methods that do not involve the determination of a compressed data representation. One promising method is [26], in which a super-resolution GAN and deep physical model are used to map low resolution, 3D $\mathrm{N}$-body simulations to their high resolution counterparts. This is similar in concept to $[19,20]$, which used GANs to map dark matter density fields to corresponding halo number count maps and hydrodynamical quantities, respectively. Reference [27] proposes a super-resolution scheme for generating large-scale realizations of the matter density

\footnotetext{
${ }^{1}$ Comprehensive list at https://github.com/georgestein/ml-incosmology.
}

field hierarchically, treating the scalability problem separately from sample accuracy.

Three-dimensional $\mathrm{N}$-body datasets are challenging to model because each sample realization contains many degrees of freedom. In addition, the voxel PDF for each realization has large tails, with matter densities spanning several orders of magnitude. Natural image datasets, a common focus of GAN applications, also contain tails in pixel space [28], however the application in cosmology is unique because the tails of the voxel PDF often have a disproportionate impact on the relevant summary statistics.

This exploratory work identifies a number of factors important for training GANs that, from a compressed representation, produce samples that are both accurate and preserve the sample variance of the data distribution. In a controlled setting, we demonstrate the need for data scalings that preserve information in the high-density regime. Furthermore, we find that heavy-tailed distributions are more suitable priors for modeling the latent space, significantly improving the accuracy of our generated covariance and correlation matrices. Through these and other considerations, we obtain state of the art results for 3D cosmic web generation as reflected through the matter density power spectrum, a range of bispectra, and other cosmological summary statistics.

The report is structured as follows: in $\S$ II we introduce the statistical and optimization framework of GANs. We describe the GADGET-2 dataset and training procedure in $\S$ III and outline the modeling choices specific to this work. The corresponding results for single redshift and redshift-interpolated models are presented in § IV. Finally, we discuss our results in $\S \mathrm{V}$, concluding with a discussion of future directions and applications.

\section{GENERATIVE ADVERSARIAL NETWORKS}

Generative adversarial networks, originally introduced in [22], are a class of unsupervised models that learn an underlying probability distribution, which we denote $p_{\text {data }}(x)$, through adversarial training. In this paradigm, a generator $G$ attempts to produce samples that appear real to a discriminator $D$. The discriminator is tasked with distinguishing between real training samples and synthetic ones.

The standard training process, shown schematically in Fig. 1, goes as follows:

(1) The input is either a random latent vector $\mathbf{z}$ [typically drawn from a unit-normal distribution $\mathcal{N}(0, \mathbf{I})]$, or some structured input.

(2) The generator $G$, parametrized by a neural network, takes the latent vector $\mathbf{z}$ and outputs a sample $G(\mathbf{z})$.

(3) The discriminator $D$, also parametrized by a neural network, takes real samples $\mathbf{x}$ and generated samples $G(\mathbf{z})$ as input, and outputs scores $D(\mathbf{x})$ and $D(G(\mathbf{z}))$, respectively. Each score represents the degree to which the discriminator believes a given sample comes from the data distribution $p_{\text {data }}(\mathbf{x})$. 


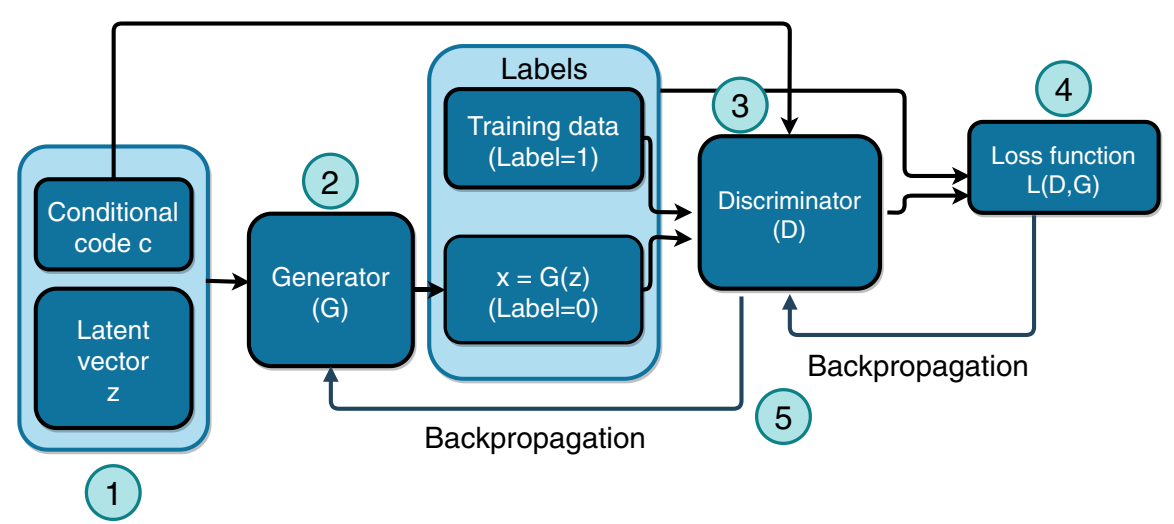

FIG. 1. Schematic for generative adversarial networks. As described in Sec. II, a latent vector is drawn from some prior (1) and used as input for the generator network (2) which outputs a sample in the data space. A batch of these samples, along with a batch of training set samples, are passed to the discriminator (3). The discriminator network outputs a set of scores representing how much it believes the samples come from the data distribution, and these scores are compared with ground truth labels to calculate a loss (4). This loss is then used to update the parameters of both the generator and discriminator network through backpropagation (5).

When the score is scaled to the range $[0,1]$, it is sometimes interpreted as an implicit likelihood of the data given the discriminative model, $p(\mathbf{x} \mid D)$.

(4) The discriminator predictions are compared to the ground truth labels, from which a loss $\mathcal{L}(D, G)$ is computed.

(5) The loss is used to update the weights of $G$ and $D$ through backpropagation, flowing through the discriminator and then through the generator.

(6) Steps 1-5 are repeated, looping through the training set over several epochs.

Formally, GAN training is a min-max problem; i.e., the generator and discriminator compete to minimize and maximize a loss function, respectively:

$$
\begin{aligned}
\min _{G} \max _{D} \mathcal{L}(G, D)= & \mathbb{E}_{\mathbf{x} \sim p_{\text {data }}(\mathbf{x})}[\log (D(\mathbf{x}))] \\
& +\mathbb{E}_{\mathbf{z} \sim p_{g}(\mathbf{z})}[\log (1-D(G(\mathbf{z})))]
\end{aligned}
$$

The first expectation on the right-hand side updates the discriminator, while the second term updates both the discriminator and the generator. By training both networks simultaneously, the generator gradually learns to produce samples from the data distribution. As described in [22], the optimal generator and discriminator reach a Nash equilibrium, where neither network can make unilateral improvement over the other. That work also demonstrated that, for an optimal discriminator and certain choices of loss function, training the generator is equivalent to reducing the Jensen-Shannon divergence between the generated distribution $p_{g}(z)$ and the empirical distribution $p_{\text {data }}(x)$. The Jensen-Shannon divergence is the symmetrized version of the Kullback-Leibler divergence:

$\mathbb{D}_{J S}\left(p_{g}(\mathbf{x}) \mid p_{\text {data }}(\mathbf{x})\right)=\frac{1}{2} \mathbb{D}_{K L}\left(p_{g}|| M\right)+\frac{1}{2} \mathbb{D}_{K L}\left(p_{\text {data }}|| M\right)$ where

$$
M=\frac{p_{\text {data }}(\mathbf{x})+p_{g}(\mathbf{z})}{2} .
$$

A common assumption when using GANs for modeling is that while the observed data may be high-dimensional, the underlying data distribution exists on a lowerdimensional manifold. In this spirit, generator outputs $G(\mathbf{z})$ may be interpreted as samples drawn from some underlying manifold $\mathcal{G}$, for which $\operatorname{dim} \mathcal{G}=|\mathbf{z}|$ and $\operatorname{Cov}(\mathcal{G})=G$, where $G$ is the neural network. In many applications, $\mathbf{z}$ is chosen to have dimension $\mathcal{O}(100)$, though other techniques like in painting and super resolution may use higher-dimensional inputs. One advantage of learning a compressed representation of the data is that generating high-dimensional samples in the data space reduces to sampling from a simple, lower-dimensional latent space and performing a forward pass through the generator network $G$. This also means that sample generation is fast, since the only computation needed after a draw from the latent distribution is a forward pass through the network.

\section{A. Conditional GANs}

There are cases where one would like to incorporate additional information relevant to the data generation process. The conditional GAN (CGAN) is a natural extension of the GAN that addresses these cases [29]. The training problem is a modified version of the original objective:

$$
\begin{aligned}
\min _{G} \max _{D} \mathcal{L}(G, D)= & \mathbb{E}_{\mathbf{x} \sim p_{\text {data }}(\mathbf{x})}[\log (D(\mathbf{x} \mid \mathbf{c}))] \\
& +\mathbb{E}_{\mathbf{z} \sim p_{g}(\mathbf{z})}[\log (1-D(G(\mathbf{z} \mid \mathbf{c})))] .
\end{aligned}
$$

Here, c denotes conditional information, which can be appended both to the latent vector of the generator and to 
the discriminator as an extra feature. A properly trained conditional generator is then able to interpolate within the conditional distribution $p(\mathbf{x} \mid \mathbf{c})$. If the marginal distribution $p(\mathbf{c})$ is known, then training the CGAN results in a model that can generate samples from the joint distribution $p(\mathbf{x}, \mathbf{c})=p(\mathbf{c}) p(\mathbf{x} \mid \mathbf{c})$. For example, in the case of the standard MNIST dataset of handwritten digits, ${ }^{2}$ an obvious conditional distribution is $c \sim \operatorname{Cat}(k)$, where $k \in\{0,1, \ldots, 9\}$. There is nothing that specifically constrains the form or dimensionality of the conditional information, so long as it can be passed through layers of the generator and discriminator.

The theoretical guarantees of GANs are compromised by a number of factors in practice. Because optimization is performed over network parameters and not over probability densities, convergence to the global optimum where $p_{g}=p_{\text {data }}$ may be complicated by critical points and/or inefficient training. Furthermore, unstable training dynamics can lead one of the networks to dominate the other such that gradients are suppressed and learning stops prematurely. When the generator dominates the discriminator, the pathology is referred to as "mode collapse," in which the generator learns to model some small subset of the data distribution. Despite these practicalities, GANs with reasonable model capacity and sufficient training data can be trained successfully and produce high-fidelity samples from the underlying data distribution [31,32].

\section{DATASET AND TRAINING PROCEDURE}

\section{A. $N$-body simulations with GADGET-2}

While many types of cosmological data have nonlinear properties, we choose to train a GAN to model the dark matter component of the "cosmic web" [33]. The cosmic web is a combination of baryonic and dark matter that evolves with the expansion of the universe and eventually clusters, forming the overdense seeds of galaxy and star formation. Due to properties of gravitational collapse in an expanding universe, the cosmic web is comprised of many coherent structures, such as one-dimensional filaments and two-dimensional sheets.

Our training set comes from a suite of thirty-two dark matter only simulations made with the software GADGET$2{ }^{3}$ Each simulation is initialized with a unique seed, using the cosmological parameters that match the best-fitting results of the WMAP + BOSS DR9 analysis [35]. The initial conditions are derived using second-order Lagrangian perturbation theory at a starting redshift of $z=49$. Each simulation volume has $512^{3}$ particles in a $(512 \mathrm{Mpc})^{3}$ volume that are evolved to the present day, with snapshots written to disk at $z=\{3.0,2.0,1.5,1.0,0.5,0.25,0.0\}$, after which they are placed on a 3D grid with 512 resolution

\footnotetext{
${ }^{2}$ See Ref. [30].

${ }^{3}$ See Ref. [34].
}

elements per side. After gridding, the GADGET-2 density field is given in units of $10^{10} M_{\odot} /$ cell, and we will refer to this normalized matter density field as $\tilde{\rho}(x)$.

A naive application of GANs to directly generate $512^{3}$-voxel samples would be challenging because of the high dimension of the data and the availability of training samples. Instead, we train on $(64 \mathrm{Mpc})^{3}$ subvolumes, which gives us a training set with 16384 samples. We further exploit the isotropy of our samples to augment our dataset through random rotations and flips, which increases the effective training set size by a factor of 6 (sides $) \times$ $4(90$ deg. rotations $) \times 2$ (random flips $)=48$.

\section{B. Network architecture}

We base our network architecture on [36], in which the generator upsamples a latent vector $\mathbf{z}$ with $3 \mathrm{D}$ transpose convolutional layers and the discriminator downsamples with 3D convolutional layers. This is one example of a deep convolutional GAN (DCGAN [37]). The parameters and activations used to configure the network are shown in Table I.

While a variety of large-scale, sophisticated neural network architectures have been presented in the machine learning literature, we found that (relatively) smaller networks had sufficient capacity to generate high-fidelity samples for our application and were also easier to train. In particular, our results did not change significantly when the dimension of the latent space was increased, when the number of filters was increased, or when extra convolutional layers were added beyond the base architecture.

\section{Data scaling}

The evolved matter density field, $\tilde{\rho}(x)$, exhibits a dynamic range spanning several orders of magnitude. While not strictly prohibited, directly generating samples with values in this range would be difficult for a generator network. In fact, [37] argues that a bounded final activation allows the model to more quickly saturate the range of the training distribution. We use the transformation from [24],

TABLE I. Architecture specifications for each layer of the generator network $(\mathrm{G})$ and discriminator network (D). Asterisk signifies that order of layers is reversed in the discriminator.

\begin{tabular}{lc}
\hline \hline Filter sizes & $\{4,4,4,4,4\}$ \\
$n_{\text {filter }}$ & $\{256,128,64,32,1\}^{*}$ \\
Strides & $\{1,2,2,2,2\}^{*}$ \\
Padding: & $\{0,0,0,0,1\}^{*}$ \\
Layer Act. & Leaky ReLU (G), ReLU (D) \\
Final Act. & Tanh $(\mathrm{G})$, Sigmoid (D) \\
Latent dimension & 100 \\
$N_{\text {params }}$ & $4,393,920$ \\
\hline \hline
\end{tabular}




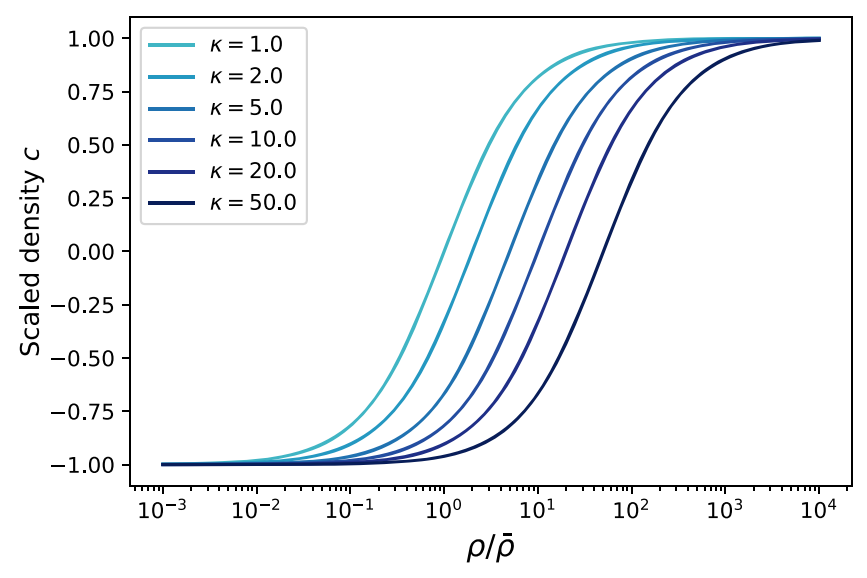

FIG. 2. Scaling from Eq. (5) plotted for different values of $\kappa$. Scalings with small values of $\kappa$ have smaller gradients at high density.

$$
c(x)=\frac{2 \tilde{\rho}(x)}{\tilde{\rho}(x)+\kappa}-1,
$$

which scales our density fields to the range $[-1,1)$, suitable for a generator network with a hyperbolic tangent $(\tanh )$ final activation.

In [24], the scaling parameter $\kappa$ is set to 4 . However, after performing a series of experiments, we choose to adopt a larger value, $\kappa=45$, that better preserves information in the high-density regime and results in more accurate samples. Figure 2 illustrates how a small variation in scaled density can correspond to a large variation in the final matter density (see Appendix B for more detailed analysis). We experimented with the logarithmic scaling used in [25] as well as other piecewise scalings, but found none were able to capture the statistics of the matter density field as well as Eq. (5).

\section{Modeling the latent space with a heavy-tailed prior}

The dynamic range of voxel matter densities and of the matter density PDF make $N$-body simulations a unique dataset. While many datasets have non-Gaussian tails in their pixel/voxel distributions, cosmological $N$-body datasets are particularly challenging to model because the least frequent, high-density voxels have the largest impact on the relevant summary statistics. For the dataset considered in this work, the challenge is in capturing the mean voxel distribution function at high density while also capturing its sample variance.

The standard approach for sample generation with a GAN is to draw a noise vector $\mathbf{z}$ from a Gaussian or uniform distribution. However, there is no strong motivation to use these as priors (beyond ease of sampling) if they do not reflect important features of the data. As shown in Sec. IV, it is only when a heavy-tailed prior is used that both accuracy and sample variance are captured by our model. To date, and to our knowledge, there are not many instances in the machine learning literature where heavytailed distributions are used to model the latent space. Reference [38] develops nonparametric priors through onthe-shelf optimization tools in order to improve latent space interpolation, though our work is less focused on semantic interpolation than common applications where natural image datasets are modeled. We choose a Student's $\mathrm{t}$-distribution prior for the latent space and tune $\nu$, the number of degrees of freedom in the distribution. The univariate Student's t-distribution has probability density

$$
P(z)=\frac{\Gamma\left(\frac{\nu+1}{2}\right)}{\sqrt{\nu \pi} \Gamma\left(\frac{\nu}{2}\right)}\left(1+\frac{z^{2}}{\nu}\right)^{-\frac{\nu+1}{2}}
$$

where $\Gamma(\cdot)$ is the Gamma function. When $\nu$ is smaller, the Student's t-distribution has larger tails, and in the limit where $\nu \rightarrow \infty, P(z)$ converges to a Gaussian distribution. Through empirical tests, we find $\nu=10$ performs best for our dataset. We reiterate that our argument for modeling the latent space is not specific to the Student's t-distribution. One might capture tail behavior with a Lorentzian distribution, a Cauchy distribution, or even a mixture of Gaussians.

\section{E. Training procedure}

The generator and discriminator were trained for 150 epochs with learning rates of $1 \times 10^{-4}$ and $5 \times 10^{-5}$, respectively, using the ADAM optimizer [39]. We chose to train with a batch size of 16 , meaning gradient updates to the networks at each iteration are computed based on 16 real and 16 generated samples. Within each epoch, each training set mini-batch is chosen randomly, after which a set of random rotations and flips are applied to the samples. Following the training procedure from [36], the discriminator's weights are only updated in a given batch iteration if its accuracy for that batch falls below $80 \%$. This is one heuristic to prevent the discriminator from outperforming the generator, and in our case led to more stable training.

We also conducted several experiments with the Wasserstein GAN [40] with gradient penalty [41] scheme (WGAN-GP). The WGAN-GP trained stably for a range of architectures, optimizers and hyperparameters. While the resulting models were able to capture the mean summary statistics of the data as described in Sec. IV, they struggled to capture sample variance in the generator distribution. Because of this, we chose to use the standard GAN for the remainder of our experiments.

After some training, a GAN may be near its optimum, but if the learning rate is too large then the networks will be limited in their ability to further converge. With this in mind, we use a learning rate scheduler that periodically reduces the learning rate by a factor $\gamma$. We choose to decay the learning rates of both the generator and discriminator by $50 \%(\gamma=0.5)$ after every 1000 gradient updates to the generator. 


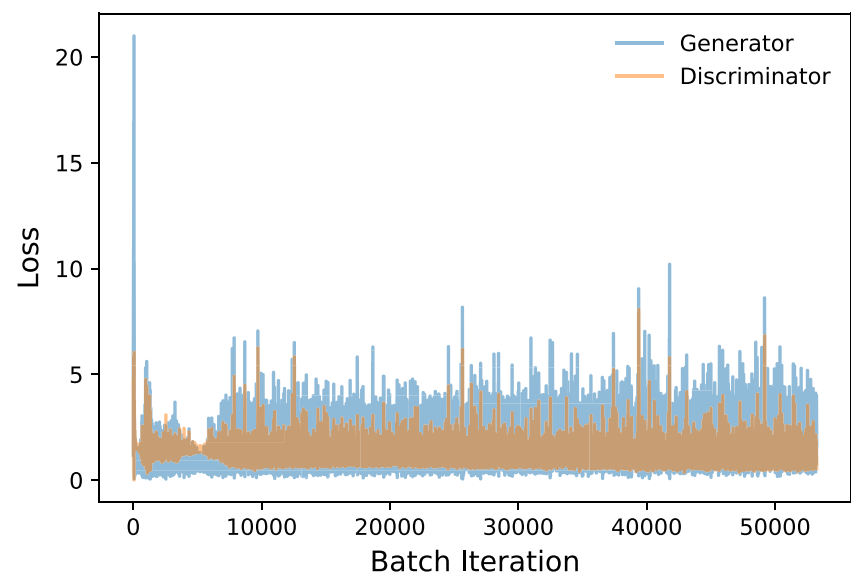

FIG. 3. Losses of the generator (blue) and discriminator (orange) as a function of batch iteration for a training run using a 3D DCGAN. The generator loss is computed from the second term in the objective from Eq. (1), while the discriminator loss is the sum of the first and second terms. The losses do not indicate when the models have converged, but help verify that the training dynamics of the GAN are controlled.

The losses of the generator and discriminator are plotted as a function of batch iteration in Fig. 3. Despite small fluctuations that come from noisy estimates of the loss computed from minibatches, training stabilizes relatively quickly. The losses do not indicate when the models have converged, but help verify that the training dynamics of the GAN are controlled. As there are no asymptotic convergence guarantees when training GANs, one is at liberty to choose the model at any point in training when it best captures the desired summary statistics. To optimize our model selection, we save a version of the generator at the end of each epoch and determine for which model the mean voxel PDF and power spectra match the data best. As discussed in Appendix A, we find a correspondence between the variance of the latent distribution and the mean power spectral amplitude of generated samples. After training, we use this to further calibrate the model to the training set by perturbing the scale width of the latent distribution, typically at the percent level.

Computations were done on the Texas Advanced Computing Cluster on a node with $4 \times$ Nvidia 1080-TI GPUs with $128 \mathrm{~GB}$ of RAM, and took roughly nine hours in wall clock time to train. The model and training were implemented using the PYTORCH library. ${ }^{4}$ We have made the code for this work publicly available on Github. ${ }^{5}$

\section{RESULTS}

We first present the results of our model trained on the full $N$-body dataset evolved to $z=0$ (present day). In this

\footnotetext{
${ }^{4}$ See Ref. [42].

${ }^{5}$ See Ref. [43].
}

section, the performance of our models is evaluated directly on the standard cosmological summary statistics. These are more physically interpretable than discriminator-based metrics such as the Frechet inception distance or the inception score that are common in the machine learning literature. For $z=0$, the model is trained on data that are scaled using (5) with parameter $\kappa=45$. In our experiments, $\kappa=45$ was the largest value where training was stable. Unless specified otherwise, our results use $N_{s}=2000$ samples to evaluate each GAN model and the full training set for GADGET-2 $\left(N_{s}=16384\right)$.

Figure 4 shows visualizations of $(64 \mathrm{Mpc})^{3}$ volumes from GADGET-2 (top row) and from a trained GAN (bottom row). Samples from the generator network contain many of the filamentary structures known to populate the cosmic web. At $(64 \mathrm{Mpc})^{3}$, samples share similar types of features but also exhibit considerable variation by eye. The large variation on these scales is expected, as the homogeneity scale of the Universe is typically accepted as $\gtrsim 100 \mathrm{~h}^{-1} \mathrm{Mpc}[44,45]$. Some periodic artifacts can be seen in synthetic samples, however these are low density features that do not significantly affect the cosmological summary statistics shown in the following subsections.

\section{A. Voxel distribution function}

Figure 5 shows voxel histograms of the scaled densities (top) and normalized matter densities (bottom) for real and synthetic samples. Both types of densities are shown to highlight the fact that while the GAN trains on data scaled between $[1,-1)$, the rescaling back to matter densities may produce unanticipated features. By using a larger scaling parameter for the data transformation, we prioritize information encoded in high-density voxels. This is reflected in the $N$-body and GAN-generated voxel PDFs, which agree well for the upper four orders of magnitude in density and diverge below $\rho / \bar{\rho} \sim 10^{-1}$. The deviation at low density is not evident when looking at the scaled voxel PDFs alone, which appear consistent across the full range.

The voxel PDFs in Fig. 5 also highlight the difference between models that use a Gaussian latent distribution and a heavy-tailed distribution. As alluded to in the previous section, initial tests produced models that generally underestimated the variance of the training set. Furthermore, this underestimated variance was present across several summary statistics. A similar phenomenon is observed and noted in [25]. If the variance of highdensity voxels is underestimated, one should expect the variance of subsequent $n$-point statistics to also be underestimated. A number of potential remedies to this problem were attempted. Adding convolutional layers to the generator/discriminator did not yield noticeable improvements, nor did modifications of the size of the latent space, the number/size of filters in the networks, the scaling of the data or the training dynamics. However, one can see that 

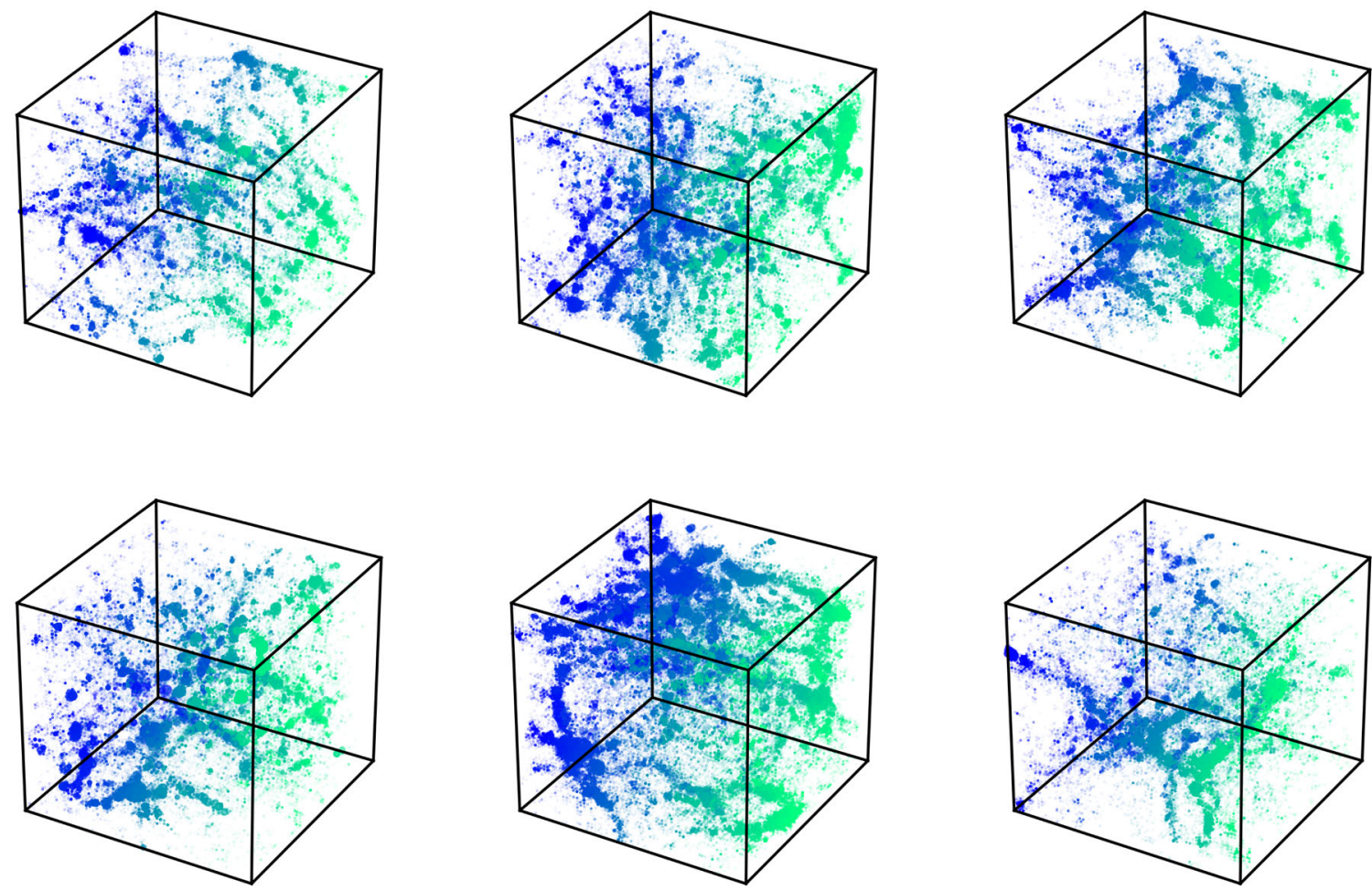

FIG. 4. 3D visualizations of GADGET subvolumes evolved to redshift $z=0$ (top row) and samples generated with a DCGAN (bottom row). Each subvolume is visualized by plotting a number of points within the volume with size $R=(c(x)+1)^{5}$, where $c(x)$ is the scaled density from (5) for a given location. This is done to highlight the relevant features of large-scale structure. Color scale denotes position along the $x$ axis.

the heavy-tailed GAN more faithfully captures variance in the high-density voxel PDF.

One validation for generative models of the matter density field is mass conservation. On the one hand, the question of mass conservation in GAN-generated simulations is ill-posed because there is no initial density field the model uses as input. However, we can examine how the distribution of mean densities $P\left(\bar{\rho}_{\text {subvol. }}\right)$ for an ensemble of generated subvolumes compares to that of $N$-body simulations. ${ }^{6}$ The average density of a sample is a weighted integral over the voxel PDF, so mismatches in the real/ synthetic voxel PDFs should lead to changes in the distribution of average densities. Figure 6 compares estimates of $P\left(\bar{\rho}_{\text {subvol. }}\right)$ from real and synthetic samples. Samples from the GAN trained with a Gaussian latent distribution have average densities $\sim 12 \%$ higher compared to samples in the training set. This is consistent with the fact that on average, the high-density voxel PDF contains more probability mass than that of the training set. The heavytailed GAN does slightly better in this regard, with an

\footnotetext{
${ }^{6}$ To clarify, $\bar{\rho}_{\text {subvol. }}$ denotes the average density of a given $(64 \mathrm{Mpc})^{3}$ subvolume, and will be different from the average density of a full GADGET-2 simulation. For the training set, each $\bar{\rho}_{\text {subvol. }}$ is computed from an already normalized matter density field $\tilde{\rho}(x)$.
}

median average density $\sim 5 \%$ higher than that of the training set.

\section{B. Power spectrum}

Figure 7 shows a comparison of real and synthetic median power spectra, along with their 16th and 84th percentiles. The power spectrum is the Fourier transform of the two point correlation function $\xi(r)$ :

$$
P(|\vec{k}|)=\int \xi(\vec{r}) e^{-i \vec{k} \cdot \vec{r}} d^{3} \vec{r},
$$

where $\xi(|\vec{r}|)=\left\langle\delta\left(\vec{r}^{\prime}\right) \delta\left(\vec{r}^{\prime}+\vec{r}\right)\right\rangle, k=2 \pi / \lambda$ is the fluctuation wave number and $\delta(\vec{r})=\rho(\vec{r}) / \bar{\rho}-1$ is the density field contrast. We use the software package NBODYKIT [46], which uses a fast Fourier transform method to compute the power spectra of our samples. Also plotted are the mean absolute fractional deviations of synthetic power spectra from GADGET-2 power spectra, i.e., $\left|\left(\bar{P}_{\text {gen }}(k) / \bar{P}_{\text {real }}(k)\right)-1\right|$.

At the physical scales considered in this work, the CDM power spectrum is highly nonlinear. In particular, $P(k)_{\text {nonlin }} / P(k)_{\text {lin }}$ grows from unity at $k \sim 0.1$ to over a factor of ten for $k \geq 1$ [47]. Both GANs produce samples that are accurate well into the nonlinear regime. However, the heavy-tailed GAN produces more accurate power spectra, with significant improvement at large $k$. 

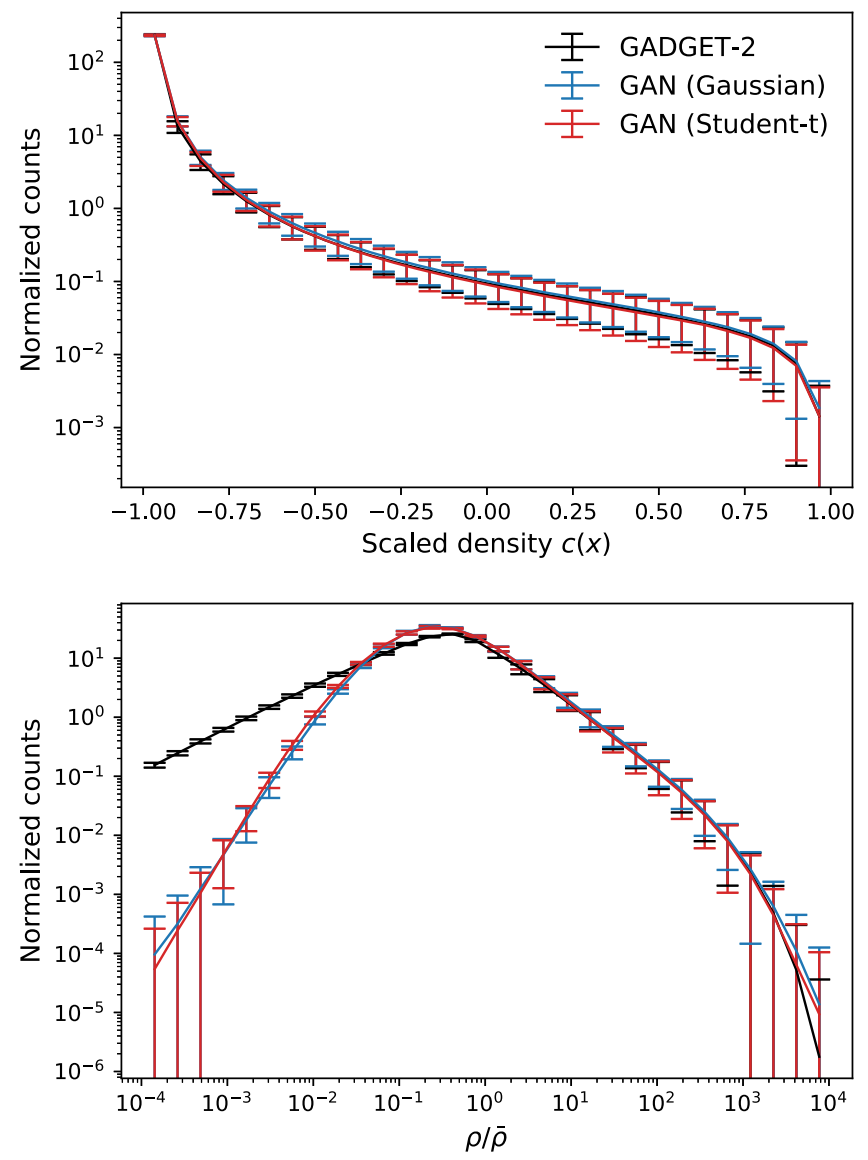

FIG. 5. Voxel probability distribution functions for scaled densities $[-1,1)$ (top) and original matter densities (bottom) of $z=0$ GADGET-2 (black) and GAN-generated subvolumes. Blue error bars correspond to a GAN model trained with a Gaussian latent space prior, while red error bars indicate results using a Student's t-distribution prior. Each set of error bars indicates the $1 \sigma$ standard deviation within each bin, computed from 2000 samples.

In particular, the mean power spectra from GADGET-2 and our heavy-tailed GAN agree within $5 \%$ for $k \leq 3$ and to $<10 \%$ up to $k=5$. At these scales, baryonic effects have a significant impact on the matter power spectrum, and so we do not claim to capture the full matter power spectrum here. Nonetheless, our results on dark matter only simulations demonstrate the ability of our generative models to capture highly nonlinear behavior on small scales. The ensemble of power spectra also captures super-sample variance from modes larger than $64 \mathrm{Mpc}$, since our training samples are subvolumes from larger simulations.

\section{Cross-power spectrum}

Cross-power spectra help quantify the degree to which generated samples are correlated with one another and with the training set at different spatial scales. Figure 8 shows $N$-body $\times N$-body, $N$-body $\times$ GAN, and GAN $\times$ GAN

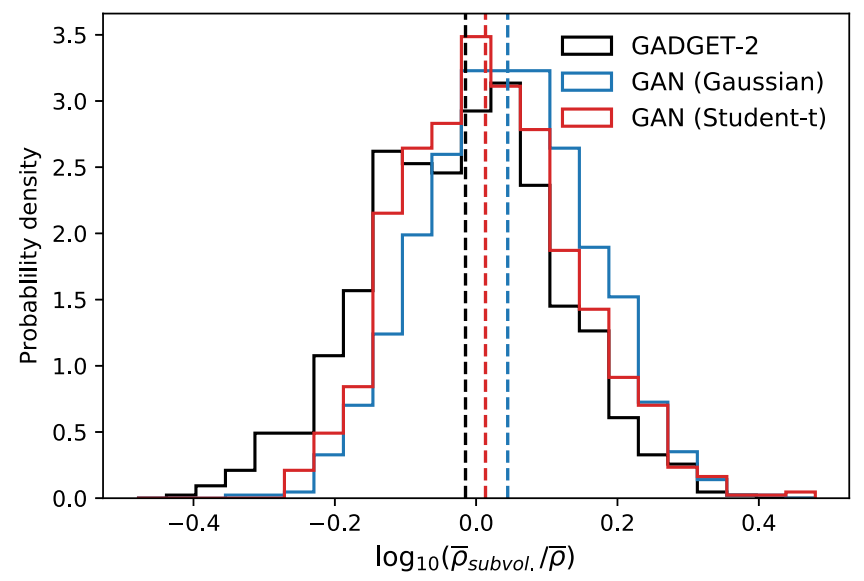

FIG. 6. Log-scaled average matter densities for $z=0$ GADGET-2 subvolumes (black) and samples generated from GANs with Gaussian (blue) and Student's t-distribution (red) latent space priors. Dashed lines indicate the median average density of each distribution. Matter density PDFs are each calculated from 2000 samples.

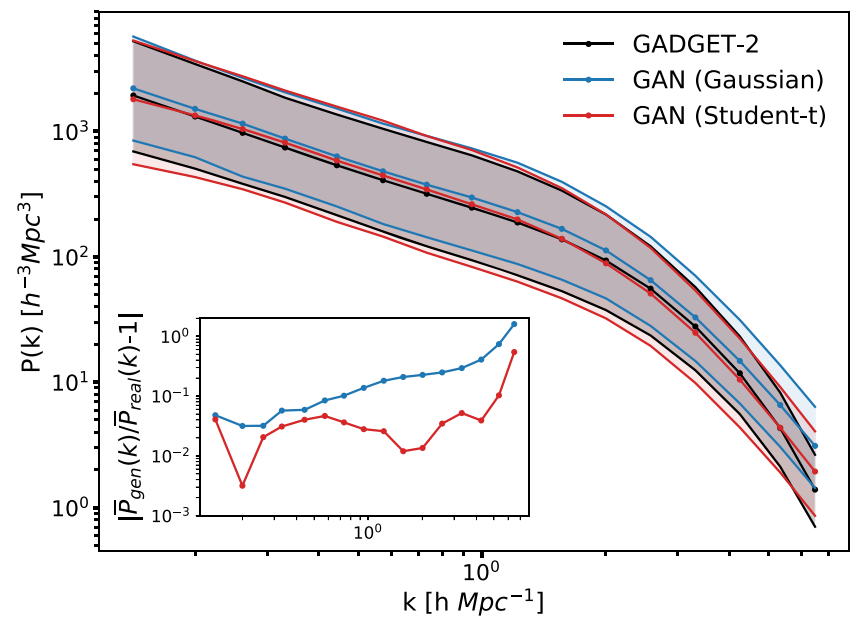

FIG. 7. Comparison of (log-scaled) power spectra between GADGET-2 subvolumes at $z=0$ (black) and samples drawn from GANs with Gaussian (blue) and Student's t-distribution (red) latent space priors. Shaded regions bound the 16th and 84th percentiles of the sample ensembles for each $k$ bin. These percentiles are calculated from 2000 samples by each GAN model and from the full GADGET-2 dataset. Bottom left corner: fractional deviations of mean power spectra between GAN and GAGDET-2 samples.

cross spectra, each evaluated from 2000 pairs of samples. Setting aside any large-scale effects that might come from evaluating subvolumes of the same $N$-body simulation, a random $N$-body sample should not (statistically) have any aligned structure with another random sample; i.e., the average cross spectrum should be zero. While the cross spectra between real and synthetic samples are consistent 


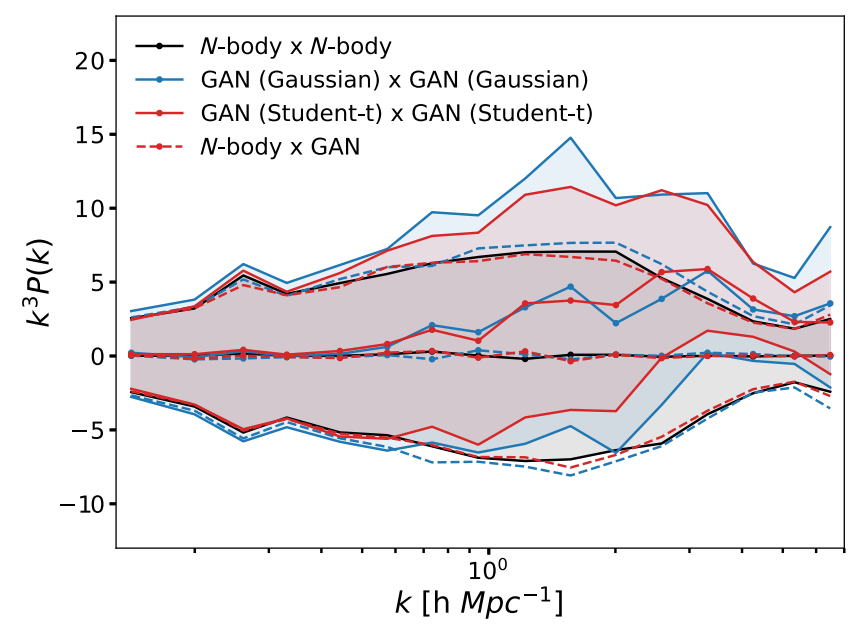

FIG. 8. Cross-power spectra for various combinations of GADGET-2 (black) and synthetic (blue, red) $N$-body subvolumes. Dashed lines indicate cross power spectra between GAN generated samples and GADGET-2 subvolumes. Each crossspectrum combination is evaluated using 2000 subvolume pairs. Shaded regions bound 16th and 84th percentiles of for each sample ensemble and within each $k$ bin. with zero, the nonzero GAN $\times$ GAN cross spectra observed for $k \geq 0.5$ indicate that our models produce some coherent structure across samples on these scales.

\section{Covariance and correlation matrices}

Figures 9 and 10 show the covariance and correlation matrices for samples generated by Gaussian and heavytailed GANs, respectively, along with their deviation from GADGET-2 $N$-body samples. We compute power spectrum covariance matrices with the sample covariance estimator

$\hat{\mathcal{C}}_{i j}=\frac{1}{N_{s}-1} \sum_{n=1}^{N_{s}}\left[P_{n}\left(k_{i}\right)-\bar{P}\left(k_{i}\right)\right]\left[P_{n}\left(k_{j}\right)-\bar{P}\left(k_{j}\right)\right]$,

in which $N_{s}$ is the number of samples, $P_{n}(k)$ is the dark matter power spectrum of sample $n$ and $\bar{P}(k)$ is the mean power spectrum across samples. The correlation matrix is further defined as

$$
\hat{R}_{i j}=\frac{\hat{C}_{i j}}{\sqrt{\hat{C}_{i i} \hat{C}_{j j}}} .
$$
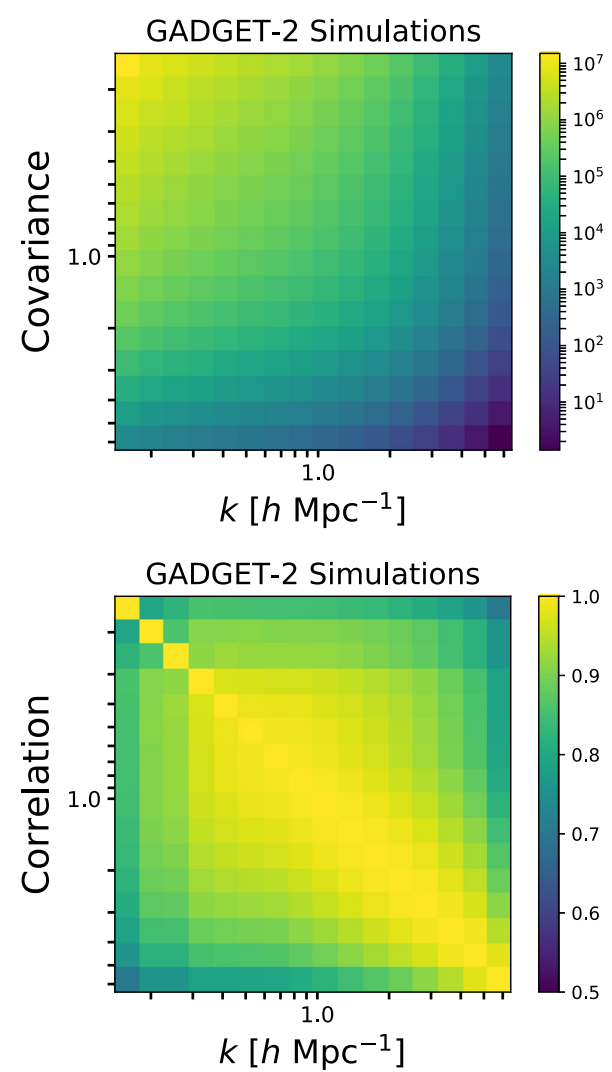
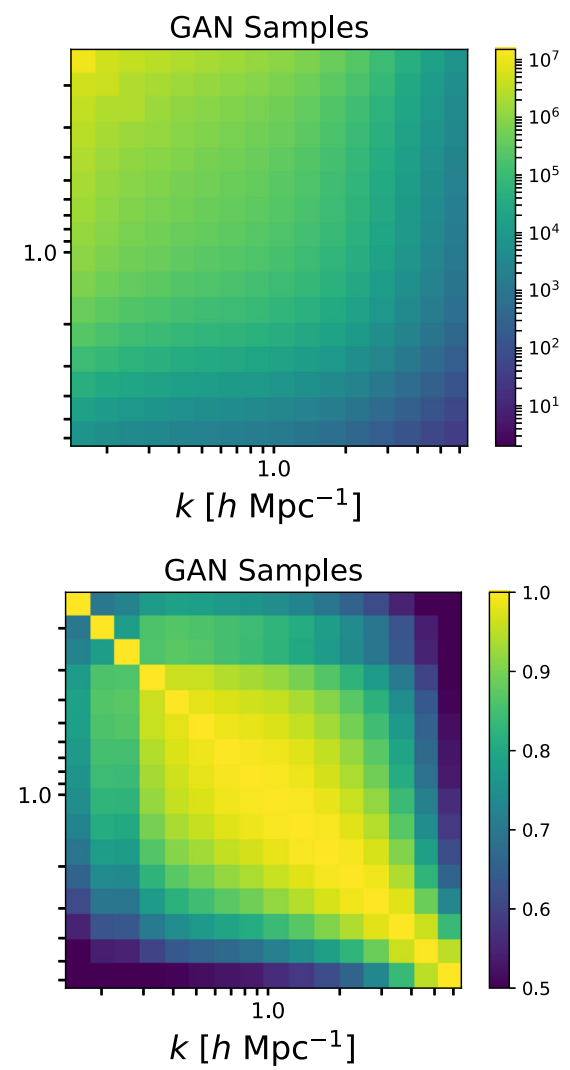
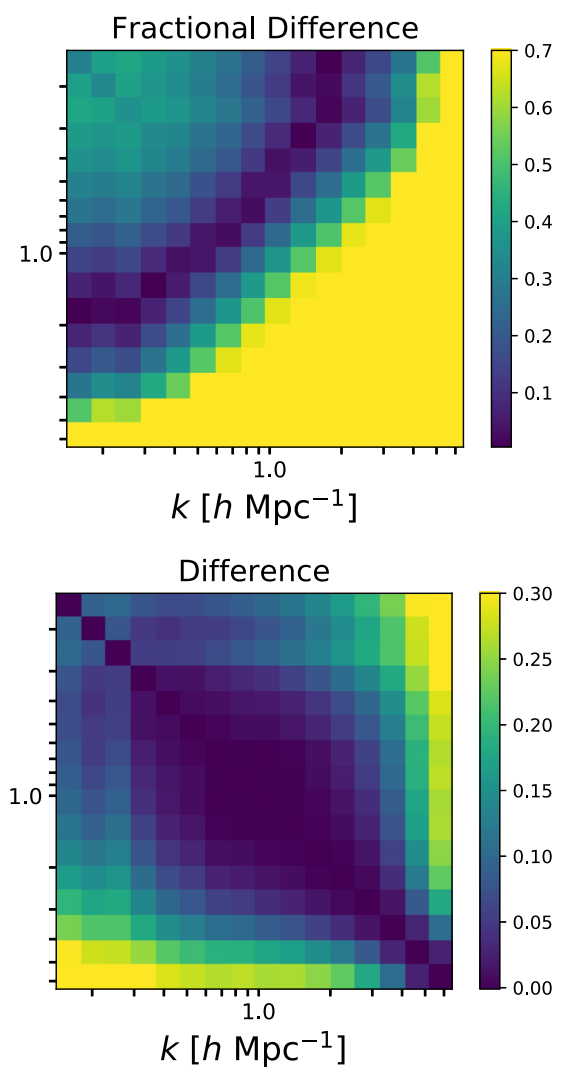

FIG. 9. Top row: power spectrum covariance matrices of GADGET-2 N-body subvolumes (left), synthetic samples drawn from a GAN with Gaussian latent space prior (middle), and their fractional difference (right). Bottom row: same as top row but for power spectrum correlation matrices and their difference. The covariance and correlation matrices from our GAN model are computed from 2000 synthetic samples, while those of GADGET-2 are computed using the full training set. 

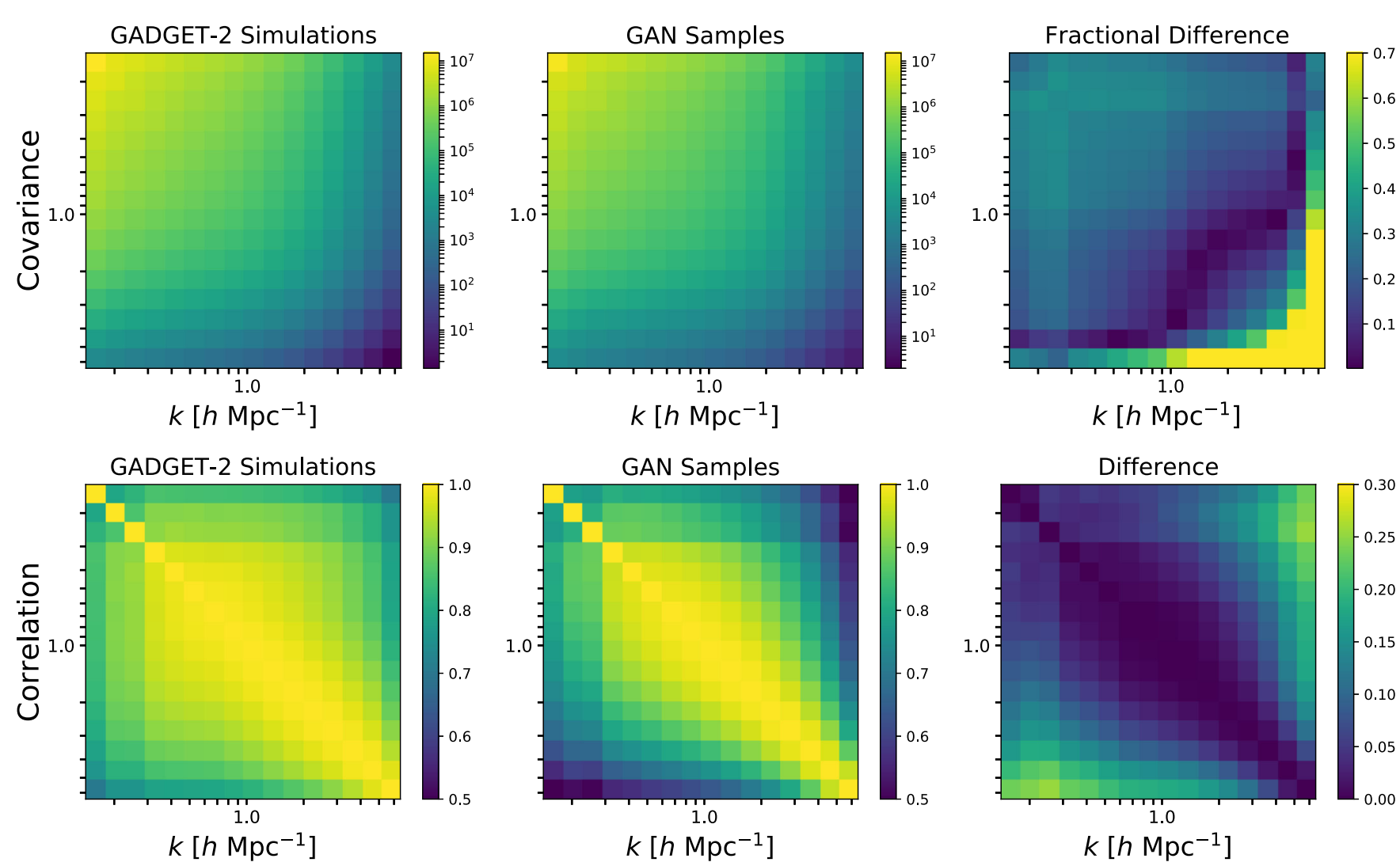

FIG. 10. The same as Fig. 9, but for a GAN where the latent space prior is modeled with a Student's t-distribution.

The improvement from using a heavy-tailed prior is especially apparent when comparing covariance matrices. The covariance matrices computed from the heavy-tailed GAN are over $50 \%$ more accurate on all scales, agreeing to between 5\%-25\% up to $k=3$. The heavy-tailed GAN also improves the estimated correlation matrices on all scales, with notable improvement for correlations between large and small scales. At high $k$, covariance matrices from both GANs overestimate the true covariance, which is consistent with overestimates of the small-scale power spectrum seen in Fig. 7. Both sets of synthetic correlation matrices follow the structure of the $N$-body correlation matrices. This is the first work to present power spectrum covariance matrices of GAN-generated 3D dark matter density fields.

\section{Bispectrum}

A comparison of matter bispectra allows us to verify that our trained GANs learn to accurately model nonlinear features of the cosmic web. The bispectrum is defined as the Fourier transform of the three-point correlation function:

$(2 \pi)^{3} B\left(\vec{k}_{1}, \vec{k}_{2}, \vec{k}_{3}\right) \delta_{D}\left(\vec{k}_{1}+\vec{k}_{2}+\vec{k}_{3}\right)=\left\langle\delta\left(\vec{k}_{1}\right) \delta\left(\vec{k}_{2}\right) \delta\left(\vec{k}_{3}\right)\right\rangle$ where $\delta_{D}$ is the Dirac delta. Figure 11 shows bispectra calculated from real and synthetic $N$-body samples, for a variety of large- and small-scale triangle configurations. We calculate sample bispectra using the software package PYLIANS. ${ }^{7}$ The accuracy of the median generated bispectra is best at small scales and gets slightly worse for larger triangle configurations (in physical space), though notably these errors appear constant with respect to $\theta$, the angle between wave vectors. The angular dependence of each bispectrum configuration also appears to be captured by the 16th and 84th percentiles of the GAN sample ensembles. Overall, the heavy-tailed GAN yields a more accurate bispectrum distribution across samples, consistent with our earlier comparisons of matter power spectra and voxel density distributions.

\section{Interpolation over redshift}

In addition to single redshift sample generation, we consider GAN models that interpolate in a latent space augmented by conditional redshift information. In particular, we seek to establish that GANs can smoothly interpolate in redshift space for a given volume. For this task, we train on snapshots of $24(512 \mathrm{Mpc})^{3}$ GADGET-2 volumes at redshifts $z=3.0,1.5,0.5$, and 0.0 , which gives us $\sim 48000$ training samples. While the scaling parameter

\footnotetext{
${ }^{7}$ See Ref. [48].
} 

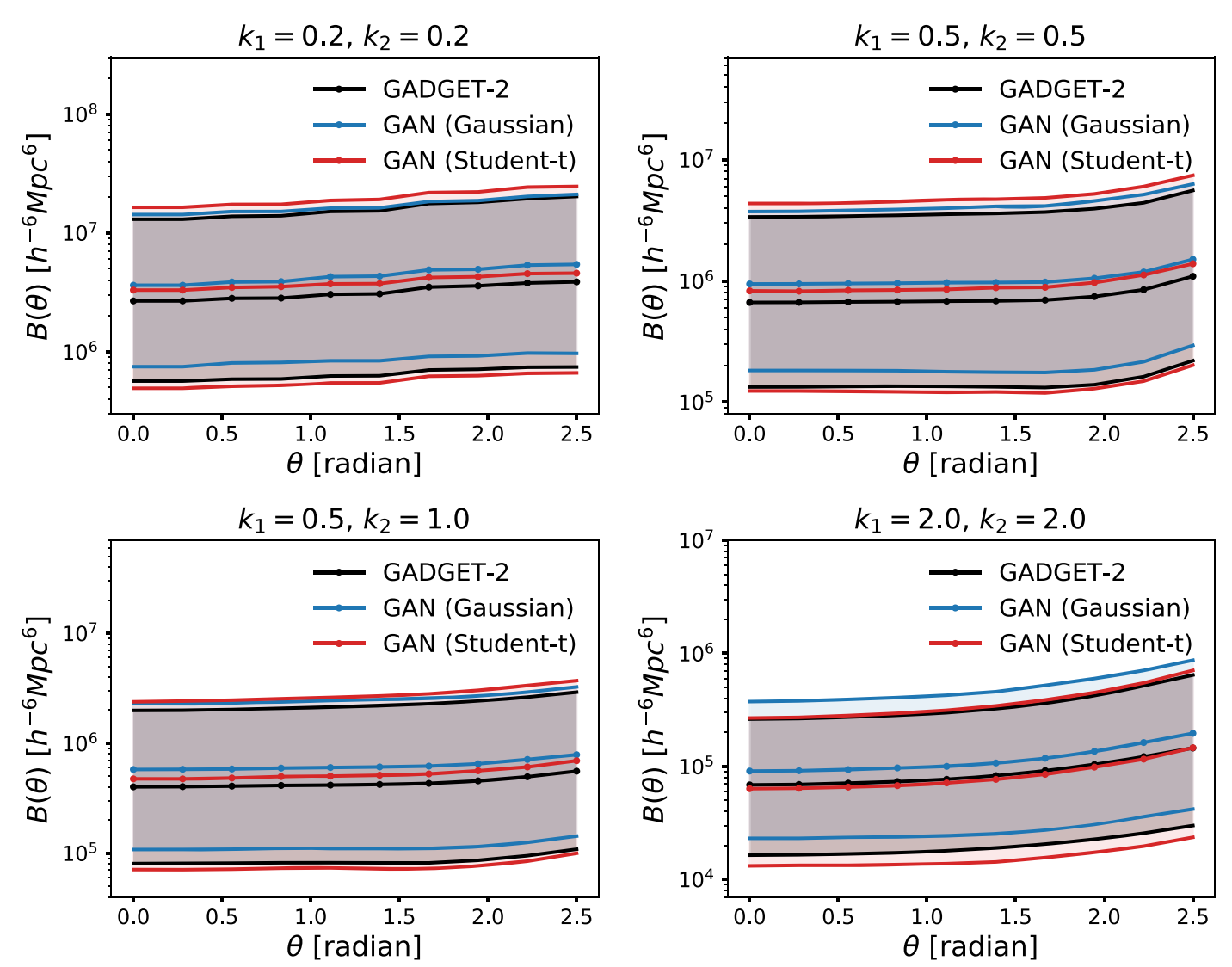

FIG. 11. Comparison of real (black) and synthetic (blue, red) matter bispectra for isosceles and other triangle configurations. Central lines indicate ensemble medians, while shaded regions bound the 16th and 84th percentiles for each set of bispectra. These percentiles are calculated from 2000 samples for each GAN model and from the full GADGET-2 dataset.

$\kappa=45$ worked well for $N$-body simulations at $z=0$, such a scaling does not effectively capture information at higher redshifts, when the matter density field is closer to Gaussian. Indeed, attempts to train our conditional redshift model with $\kappa=45$ were unsuccessful and often ended in divergent training dynamics. We instead use $\kappa=4$, which allows for stable training, albeit at the cost of accuracy on small scales.

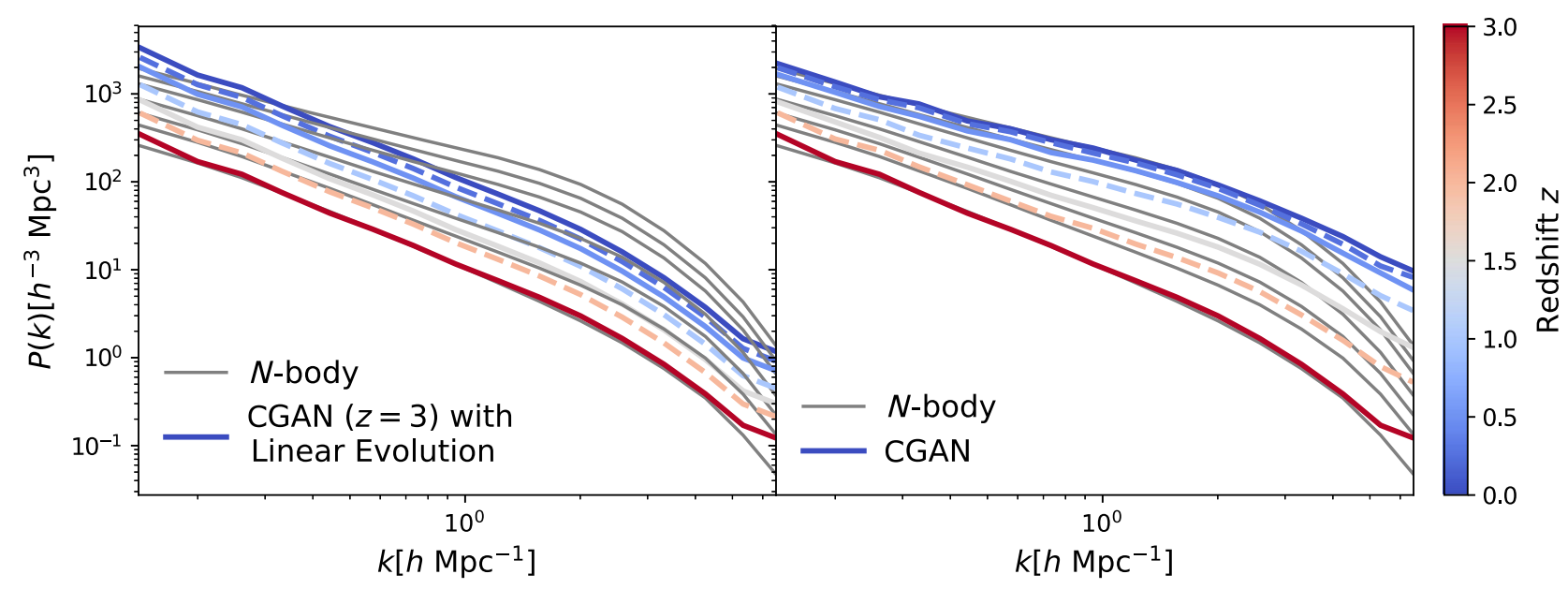

FIG. 12. Left: $N$-body power spectra (gray lines) compared with a median initial CGAN power spectrum at $z=3$ that is evolved with linear theory (colored lines). Right: the same $N$-body power spectra, compared with CGAN-interpolated median power spectra. Power spectra in both panels are evaluated at both training redshifts $(z=3.0,1.5,0.5,0.0$, solid lines $)$ and interpolated redshifts $(z=2.0,1.0$, 0.25 , dashed lines), with color bar denoting the conditional redshift input to the CGAN. 

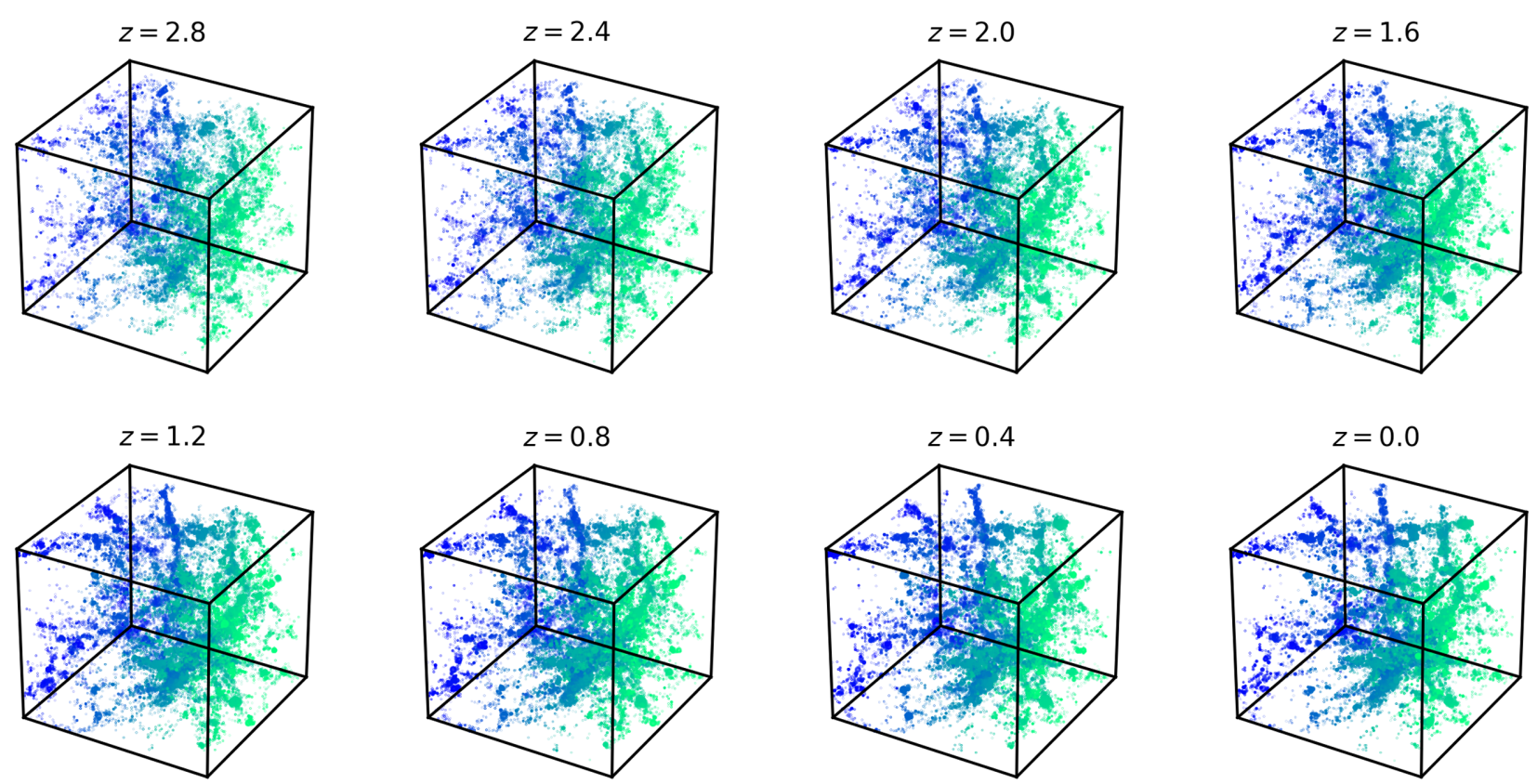

FIG. 13. 3D visualizations of CGAN-generated subvolumes, with conditional redshift $z$ linearly interpolated from $z=2.8$ to $z=0$. Subvolumes are visualized using the same scheme as in Fig. 4.

Figure 12 shows the extent to which our conditional GAN learns a nonlinear mapping between redshift and the output density field. In the left panel, an initial power spectrum generated by the conditional GAN at $z=3$ is evolved linearly,

$$
P(k, z)=\left(\frac{D(z)}{D(z=3)}\right)^{2} P(k, z=3)
$$

where $D(z)$ is the linear growth factor, while in the right panel the CGAN-interpolated matter power spectra are shown. We evaluate our model at both the aforementioned training redshifts and at intermediate redshifts $z=2.0,1.0$, and 0.25 . It is clear that while linear theory underestimates the growth of structure on small scales, the conditional GAN successfully captures the nonlinear gravitational evolution. There is no obvious difference in CGAN performance evaluated at trained vs interpolated redshifts, though interpolated power spectra at low redshift $(z<1)$ appear to be clustered around $z=0.25$.

Figure 13 shows a sequence of CGAN-generated samples where the latent vector $\mathbf{z}$ is fixed and the conditional redshift parameter is varied from $z=2.8$ to the present day. Because we do not present consecutive redshift snapshots of the same volume to the discriminator and because we do not enforce mass conservation when varying the latent redshift parameter, we do not expect the CGAN to learn to simulate gravitational evolution per se, which would involve accurate modeling of the matter density displacement field. Nonetheless, our model learns a smooth mapping between redshift and the matter density field in real space-regions that are mildly clustered at high redshift appear to form coherent filaments as the conditional redshift is varied, with no obvious intermediate discontinuities. This visual inspection helps establish the property that smooth paths in the latent space correspond to smooth paths in the data space, which reinforces the robustness of the compressed GAN representation.

\section{DISCUSSION AND FUTURE WORK}

In this work we have demonstrated that generative adversarial networks can learn a high-fidelity, compressed representation of the 3D dark matter density field. Once the generative model is trained, new samples can be generated in a fraction of a second-including the overhead from memory transfer between GPU and CPU, generating 512 samples (the volume equivalent of one $N$-body simulation) takes $\sim 6$ seconds. Furthermore, our trained generator only takes $17 \mathrm{Mb}$ of storage, and storage of individual realizations amounts to saving latent vectors that can be passed through the generator when needed. We exploit the rotational invariance of our cosmological $\mathrm{N}$-body simulations by using random rotations and flips to augment the training dataset, which contributes to the stability of training. In fact, we are able to stably train our GANs with as few as 2048 samples (see Appendix C), equivalent to four $(512 \mathrm{Mpc})^{3}$ GADGET-2 simulations.

Synthetic samples were validated using one-, two-, and three-point statistics that are accurate well into the nonlinear regime where baryonic effects become important. This work presents the first estimates of power spectrum covariance matrices and bispectra for GANs sampling from 
a compressed representation of the data. While Fig. 8 indicates some coherent structure in synthetic samples on small scales, our models largely reproduce the power spectrum covariance structure of the matter density field when compared with $N$-body simulations.

Given the dynamic range of voxel densities recovered from $N$-body simulations, recovering accurate realizations over the full, unscaled voxel PDF is difficult. High density voxels have the largest impact on summary statistics like power spectra and bispectra, and so in this work we chose a data scaling that maximizes contrast in that regime. Some cosmological analyses may warrant more emphasis on low-density features (e.g., studies of the Lyman-alpha forest), in which case the data scaling can be modified to preserve information at those densities. However, it is unclear whether an arbitrary voxel PDF with large dynamic range can be learned, given the precision of a single network and the unsupervised nature of GAN training. One might train several networks on different scaled versions of the same data; however, this is beyond the scope of this work.

This work also demonstrates that conditional GANs are capable of learning a smooth mapping between early and late times in the evolution of the matter density field, with fairly accurate interpolation as seen through the dark matter power spectrum. Future implementations might use a redshift-dependent data scaling that preserves the information in linear fluctuations at high redshift and nonlinear fluctuations at low redshift, though our attempts to do so were unsuccessful.

We also show that using a Student's t-distribution prior for the latent space results in a model that better captures sample variance across several summary statistics. While it is true that deep neural networks have high capacity, a given modeling task may be harder in the presence of distributional mismatch between the latent space and the data space. A similar point is argued in [49], which considers the role of dual data representations for classification. In that work, even when networks were given sufficient capacity to learn a dual transformation from position space to momentum space, where classification was straightforward, no trained model learned one. To our knowledge, this is the first application of heavy-tailed priors for modeling the latent space of a GAN. While we choose the Student's tdistribution as our heavy-tailed prior, we remain agnostic to the "optimal" prior. Depending on the dataset and purpose of the generative model, it may be possible to optimize the prior distribution nonparametrically as in [38]. We expect this modification of the standard GAN prior to be helpful for datasets with tails that impact the overall quality and diversity of the data distribution. While heavy-tailed priors are not necessarily the only remedy for issues addressed in this work, it was the only modification among several to the fiducial model and training procedure that led to models that were accurate and variance preserving. Furthermore, the change is very simple to implement-in our work, only one line of code needed to be changed.

Because our GANs learn a compressed representation of the data distribution, it may be possible to further decorrelate synthetic $N$-body ensembles by resampling the latent space of the generator after training. When one trains a GAN to model data with a lower-dimensional latent space, the underlying assumption is that a compressed representation of the data distribution exists on a manifold with dimension equal to that of the latent space. Mapping training samples to the posterior $P\left(\mathbf{z} \mid x_{\text {data }}\right)$ is straightforward in models like variational autoencoders where the encoder network learns the mapping during training-for GANs this may be possible through a back propagation optimization scheme like in [50]. An estimate of the training set posterior could help determine which regions of the latent space are correlated. With an approximate posterior available, one might then train a normalizing flow to resample the latent space in a decorrelated manner, as has been done with variational autoencoders in [51].

A promising avenue in machine learning literature seeks to embed inductive biases into neural network models, such that they are guaranteed to respect the structures and symmetries of a system [52-54]. Such properties make generative models more robust and generalizable, and will be important for any applications of deep generative modeling to perform physical inference.

This work focuses on optimizing performance while using a fairly simple network architecture, but larger-scale applications may warrant more sophisticated schemes. If scaling up is done by stitching together subvolumes, one needs to ensure continuity at the boundary of subvolumes, and also ensure that spatial modes larger than the subvolumes are properly modeled. One implementation uses super-resolution techniques and conditional neighbor information to generate large $\mathrm{N}$-body volumes [27]. As described in Sec. IV, we experimented with simple extensions to our model, for example adding extra convolutional layers and expanding the latent space dimension, but did not see performance improvements. The issue of "going deeper" is known to be delicate in the GAN literature [32], requiring advanced regularization techniques that we do not attempt in this work, but it may be possible to optimize neural network architecture in a more detailed fashion to capture the proper correlation structures seen in $N$-body simulations.

Generally speaking, the applicability and practical utility of GAN models depend on the data quality of the training set and the interpolation accuracy of the generative model. While conditional GANs are only discussed briefly in this work (with redshift interpolation), we anticipate GANs will have significant utility as high-dimensional conditional emulators. Once larger, high-fidelity volumes can be generated, it may be possible to further condition the generator on cosmological parameters, for example 
$\Omega_{m}$ and $\sigma_{8}$. Many validation tests remain, but GANs could be integrated with larger, physically motivated simulation pipelines to bridge the gap between density fields and observables. With a sufficiently expressive and robust model, it may be possible to use GANs as signal priors that are fit directly to observational data through a sampling scheme or gradient based optimization.

\section{ACKNOWLEDGMENTS}

R.M.F. is supported by the California Institute of Technology. P. B. was supported by Jet Propulsion Laboratory, California Institute of Technology, under a contract with the National Aeronautics and Space Administration. The authors would like to thank TzuChing Chang, Olivier Doré, Michael Albergo, Jeremy Bernstein and Yun-Ting Cheng for useful discussions. The authors acknowledge the Texas Advanced Computing Center (TACC) at The University of Texas at Austin for providing GPU cluster resources that have contributed to the research results reported within this paper: Ref. [55]. The authors also acknowledge the use of the following software for visualization and analysis: H5PY, MATPLOTLIB, NBODYKIT [46], NUMPY [56], POWERBOX [57], PYLIANS, and PYTORCH.

\section{APPENDIX A: EXPLORING THE CONNECTION BETWEEN DISCRIMINATIVE AND COSMOLOGICAL BIAS}

One challenge in using generative models to make predictions is that the statistics of generated samples are typically biased with respect to the data. For example, when generating cosmological volumes we never explicitly constrain the model to generate samples consistent up to the power spectrum, bispectrum, etc. Generative adversarial networks represent a framework in which an implicit likelihood is defined by a discriminator, which coevolves with the generator-there is no tailored loss function based on summary statistics. Even if one were used, inefficient training and/or insufficient model capacity often prohibit neural networks from fully capturing a given target distribution. While the working assumption is that a trained generator will produce samples with a distribution equal to that of the true distribution, certain samples may have artifacts or excess power that make them outliers with respect to $p_{\text {data }}$. On the other hand, because sample generation with GANs is fast, we are well justified to explore bias reduction techniques that may degrade the nominal computational performance. Most bias reduction techniques in the machine learning literature cite improvement according to neural network based metrics such as the Frechet inception distance [58] and the inception score [59]. However, it is unclear whether bias reduction with respect to the discriminator corresponds to bias reduction for cosmological metrics.
One such bias reduction technique is discriminator rejection sampling (DRS) [60]. In standard rejection sampling, samples from a distribution $X$ with density $f(X)$ are drawn using samples from another distribution $Y$ with density $g(Y)$. This is done by computing $f(y) / M g(y)$, where $M$ is some finite bound on the likelihood ratio $f(X) / g(X)$. The idea behind DRS is that, rather than perform postprocessing to correct individual samples, one can correct ensemble-based expectations by training the discriminator network to learn the ratio $p_{\text {data }}(x) / p_{g}(x)$, where $p_{\text {data }}(x)$ and $p_{g}(x)$ are densities of the real and synthetic distributions, respectively. For a fixed generator, minimizing the loss of the discriminator defined by a sigmoid and trained with cross-entropy loss yields

$$
D^{*}(x)=\frac{p_{\text {data }}(x)}{p_{\text {data }}(x)+p_{g}(x)},
$$

where $D^{*}$ is the optimal discriminator. One can also express the discriminator output in terms of the logit $\tilde{D}(x)$ (again for a sigmoid activation):

$$
D^{*}(x)=\frac{1}{1+e^{-\tilde{D}^{*}(x)}}=\frac{p_{\text {data }}(x)}{p_{\text {data }}(x)+p_{g}(x)} .
$$

Rearranging terms, the ratio $p_{\text {data }}(x) / p_{g}(x)$ can be expressed as

$$
\frac{p_{\text {data }}(x)}{p_{g}(x)}=e^{\tilde{D}^{*}(x)} .
$$

The likelihood ratio bound is set to $M=$ $\max _{x} p_{\text {data }}(x) / p_{g}(x)=e^{\tilde{D}^{*}\left(x^{*}\right)}$. In practice we cannot compute the true maximum, so instead we estimate a lower bound using a large number of samples. Finally, the acceptance probability for rejection sampling can be written as $\alpha=e^{\tilde{D}^{*}(x)-\tilde{D}_{M}^{*}}$.

There are several caveats that degrade discriminator rejection sampling in practice. For one, the acceptance probability we estimate is only approximate to the true density ratio, since we cannot directly optimize over density functions. Furthermore, if the supports of $p_{\text {data }}$ and $p_{g}$ have a small intersection, then discriminator rejection sampling may yield vanishingly small acceptance probabilities. We counteract excessively small acceptance probabilities by modifying the logit $F(x)$ of the final sigmoid activation that defines the acceptance probability:

$$
\frac{1}{1+e^{-F(x)}}=e^{\tilde{D}^{*}(x)-\tilde{D}_{M}^{*}}
$$

In the above equation, $F(x)$ can be computed from $\tilde{D}^{*}(x)$ and $\tilde{D}_{M}^{*}$, but in practice we include tunable parameters $\epsilon$ and $\gamma$ :

$\hat{F}(x)=\tilde{D}^{*}(x)-\tilde{D}_{M}^{*}-\log \left(1-e^{\tilde{D}^{*}(x)-\tilde{D}_{M}^{*}-\epsilon}\right)-\gamma$, 


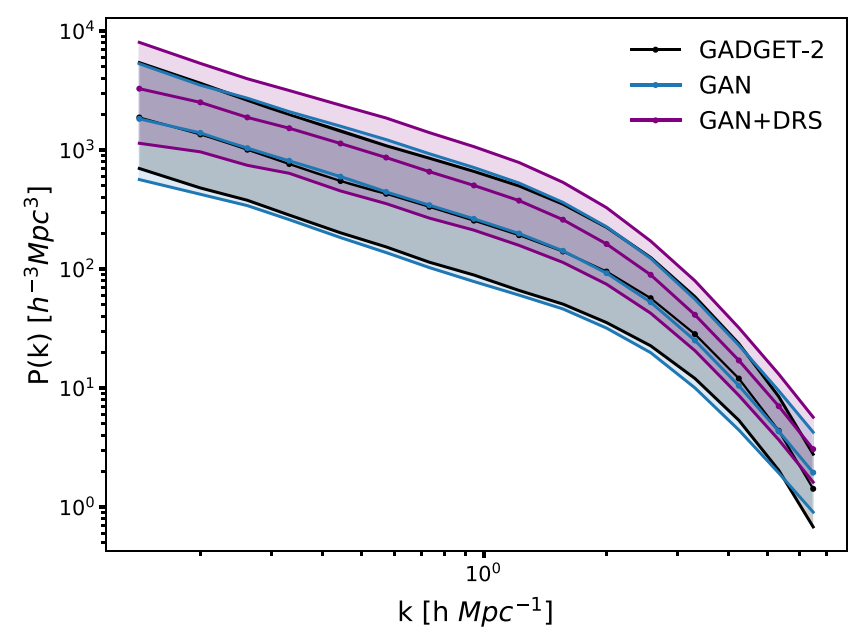

FIG. 14. Comparison of matter power spectra between GADGET-2 subvolumes at $z=0$ (black), samples drawn from a heavy-tailed GAN (blue) and samples obtained performing discriminator rejection sampling (DRS) on the heavy-tailed GAN (purple). Shaded regions bound the 16th and 84th percentiles of each ensemble and within each $k$ bin. These percentiles are calculated based on 2000 samples for each model and from the full GADGET-2 dataset.

After training the generator, we train the discriminator for an additional five epochs (with the generator fixed) such that it minimizes its loss, which plateaus around $10^{-6}$. Once this is done, we perform rejection sampling with hyperparameters $\epsilon=0.01$ and $\gamma=0.0$, which gives an acceptance rate of $\sim 3 \%$. Larger values of $\gamma$ lead to lower acceptance fractions, while more negative values yield the opposite. We calculate a lower bound for $\tilde{D}^{*}(x)$ from 2000 generated samples.

Figure 14 shows a comparison of synthetic power spectra from a heavy-tailed GAN, both with and without DRS. The choice of a relatively unbiased model serves as a null test for DRS; i.e., the accuracy of generated power spectra should remain the same or improve if DRS is effective. To the contrary, we find that the trained discriminator preferentially accepts samples with higher power on average, ironically biasing our model more. We experimented with different values of $\gamma$ and found that when gamma was higher (i.e., when acceptance probabilities were lower), the accepted samples were more biased with respect to the mean power spectrum.

While DRS is evidently impractical for our purposes, it does provide a number of important insights to this work. For one, performing DRS demonstrates that our notion of cosmological bias (in one case, bias on the amplitude of the matter power spectrum) does not align with the more generic distributional "bias" as determined by the discriminator. DRS also gives us insight into the latent space of the generator. Figure 15 shows the $\ell_{2}$-norm distribution for a set of latent vectors drawn from the prior distribution, as well as latent vectors of samples accepted through DRS.

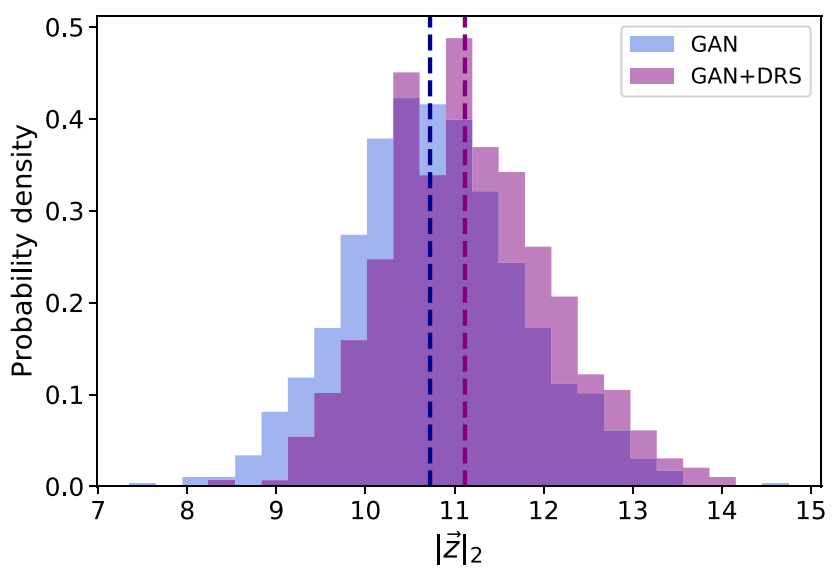

FIG. 15. Comparison of $\ell_{2}$-norm distribution for latent vectors sampled by a heavy-tailed GAN (blue) and resampled latent vectors obtained after performing discriminator rejection sampling (purple). Dashed vertical lines indicate medians of the two distributions.

One can see that DRS preferentially accepts samples drawn from latent vectors with larger magnitudes on average, while the scale dependence of the resampled power spectra remains intact. This suggests a relationship between the width of the resampled latent distribution and the power amplitude of samples. While these results are specific to the models trained in this work, DRS provides an opportunity to reason about how bias of the data distribution in discriminator-based, implicit likelihood methods compare with more physical notions of bias.

\section{APPENDIX B: SCALING PARAMETER}

In Sec. III C, we advocate for the use of a data scaling that preserves high-density features that have the most significant impact on our summary statistics. We motivate this by observing that when the scaling parameter $\kappa$ is small, the gradients in overdense regions (which can be very large in voxel space) get significantly suppressed in the scaled data. This is shown visually in Fig. 2, which has (5) plotted for different choices of $\kappa$. Capturing gradients in the high-density regime accurately with convolutions becomes more difficult, since small fluctuations in the final map may produce unrealistic values when scaled back to the original densities.

Another way to think about this problem is in terms of how a generic error gets propagated through the inverse data scaling. Recall the scaling used in this work:

$$
c=\frac{2 \rho}{\rho+\kappa}-1
$$

By computing the derivative of the inverse transformation, $d \rho / d c$, one can relate an error in $c$ to error in $\rho$ : 


$$
\delta \rho=\frac{2 \kappa}{(1-c)^{2}} \delta c
$$

For a fixed density $\rho$, the ratio of scaled values for different values of $\kappa$ is

$$
\frac{c_{1}}{c_{2}}=\left(\frac{\rho-\kappa_{1}}{\rho-\kappa_{2}}\right)\left(\frac{\rho+\kappa_{2}}{\rho+\kappa_{1}}\right) .
$$

For fixed $\rho$ and fixed $\delta c$,

$$
\begin{aligned}
\frac{\delta \rho_{1}\left(\kappa_{1}\right)}{\delta \rho_{2}\left(\kappa_{2}\right)} & =\frac{\frac{2 \kappa_{1}}{\left(1-c_{1}\right)^{2}} \delta c}{\frac{2 \kappa_{2}}{\left(1-c_{2}\right)^{2}} \delta c}, \\
& =\frac{\kappa_{1}}{\kappa_{2}}\left(\frac{1-c_{2}}{1-c_{1}}\right)^{2} .
\end{aligned}
$$

The last term in parentheses can be written as

$$
\frac{1-c_{2}}{1-c_{1}}=\frac{\kappa_{2}}{\kappa_{1}}\left(\frac{\rho+\kappa_{1}}{\rho+\kappa_{2}}\right) \text {. }
$$

Finally,

$$
\begin{aligned}
\frac{\delta \rho_{1}\left(\kappa_{1}\right)}{\delta \rho_{2}\left(\kappa_{2}\right)} & =\frac{\kappa_{1}}{\kappa_{2}}\left(\frac{\kappa_{2}}{\kappa_{1}}\right)^{2}\left(\frac{\rho+\kappa_{1}}{\rho+\kappa_{2}}\right)^{2}, \\
& =\left(\frac{\rho+\kappa_{1}}{\rho+\kappa_{2}}\right)^{2} \frac{\kappa_{2}}{\kappa_{1}} .
\end{aligned}
$$

As we are primarily concerned with the high-density regime, we can take the limit where $\rho \gg \kappa$ to conclude

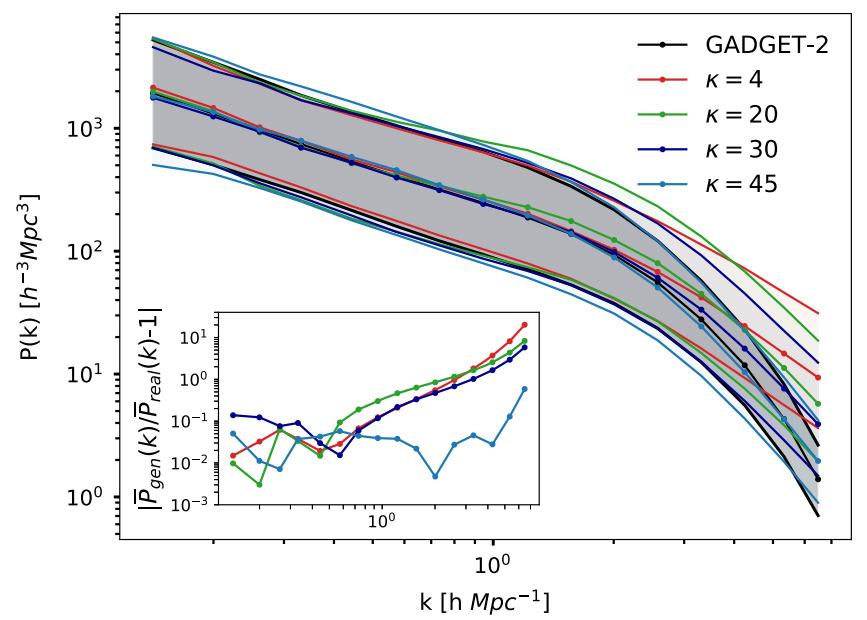

FIG. 16. Comparison of real and synthetic power spectra, varying the data scaling parameter $\kappa$ from Eq. (5) for each training run with a heavy-tailed GAN. Shaded regions bound the 16 th and 84th percentiles of the ensemble within each $k$ bin, which are calculated based on 2000 samples for each model and from the full GADGET-2 dataset. Higher values of $\kappa$ prioritize high-density features in the matter density field when the data are scaled to $[-1,1)$. that $\delta \rho_{1} / \delta \rho_{2} \approx \kappa_{2} / \kappa_{1}$. This calculation is ill posed in the sense that we have not identified specific, quantifiable uncertainties of the GAN model. Nonetheless, it demonstrates in a fairly generic setting how data scalings affect models with finite precision in reference to some target distribution.

Figure 16 shows the effect of $\kappa$ on the ability of our GANs to reproduce the power spectral distribution calculated from GADGET-2 $N$-body samples. While $\kappa=45$ produces a model that outperforms others on nearly all scales, the improvement is most significant for $k \gtrsim 1$, where the power spectrum accuracy is over a factor of 10 better than models trained with smaller values of $\kappa$. Beyond $\kappa=45$, training was unstable and the generator was unable to learn a reliable representation of the data.
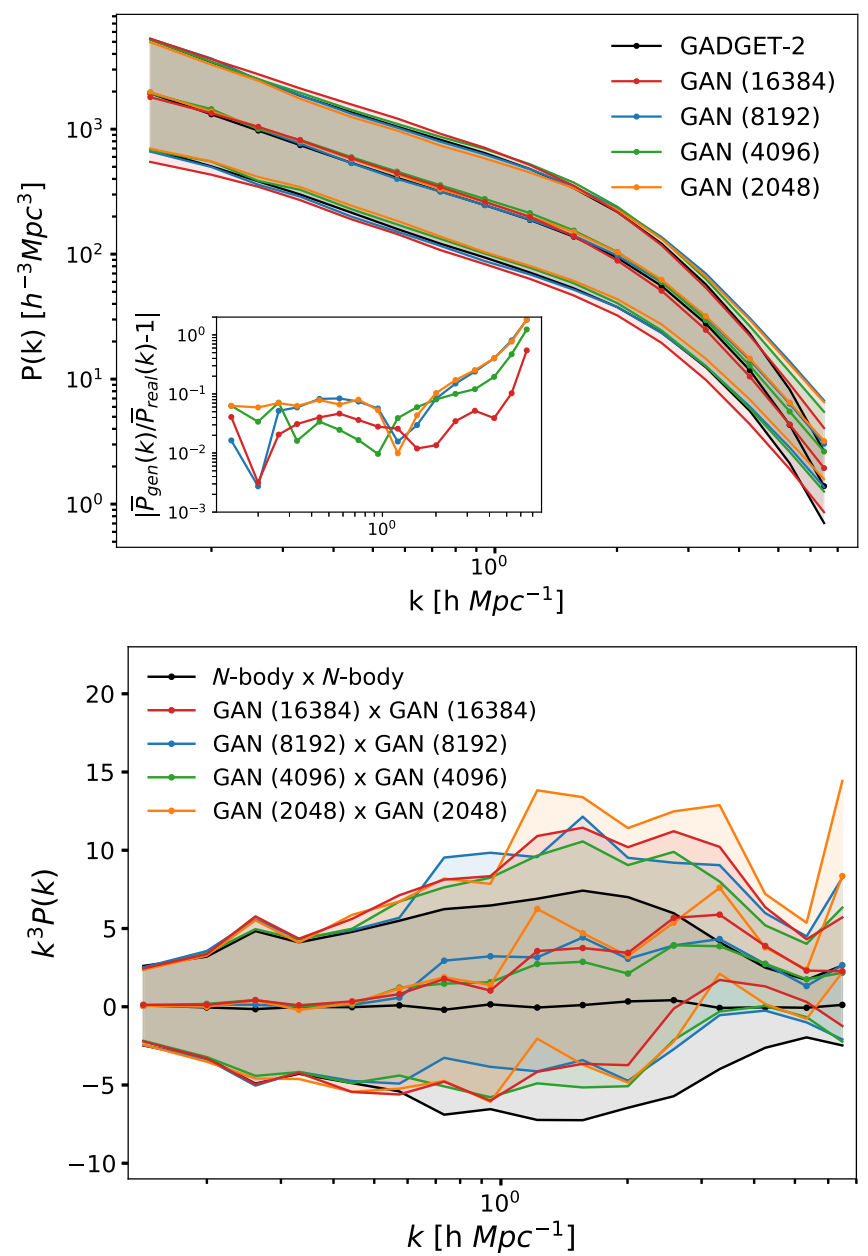

FIG. 17. Comparison of synthetic power spectra (top) and cross spectra (bottom) from GANs trained on the full training set (16384 samples), along with one-half (8192 samples), one-fourth (4096 samples), and one-eighth (2048 samples) of the full training set. Shaded regions bound the 16th and 84th percentiles of the ensemble within each $k$ bin, which are calculated based on 2000 samples for each model and from the full GADGET-2 dataset. 


\section{APPENDIX C: TRAINING DATASET REDUCTION EFFECT ON PERFORMANCE}

As data-driven models, generative adversarial networks require a large amount of training data to learn the underlying data distribution. Much like network architectures and training hyperparameters, the size and diversity of the training dataset are important factors in the stability of GAN training. For a nonstandard training set like our own, it is unclear how many training samples are needed to successfully train our GAN models.

To understand the effect of training set size on performance, we train three configurations of the same model using one-half, one-fourth, and one-eighth of the full training set. We fix all hyperparameters in the GAN across runs. While some runs on smaller training sets required restarting the training to achieve stable gradient updates in the first epoch, all models were able to train successfully. Figure 17 shows power spectra (top) and cross spectra (bottom) generated from the trained models. The power spectra generated from all three models are accurate to within $10 \%$ up to $k=2$, but none of them performs as well in the highly nonlinear regime as a model trained on the full dataset. There is no clear relationship between the training set size and power spectrum accuracy among the four models, however the model trained on the least amount of data $\left(n_{\text {train }}=2048\right)$ appears to have a larger cross-correlation on average between samples.
[1] M. Vogelsberger, F. Marinacci, P. Torrey, and E. Puchwein, Cosmological simulations of galaxy formation, Nat. Rev. Phys. 2, 42 (2020).

[2] L. Blot, P. S. Corasaniti, L. Amendola, and T. D. Kitching, Non-linear matter power spectrum covariance matrix errors and cosmological parameter uncertainties, Mon. Not. R. Astron. Soc. 458, 4462 (2016).

[3] S. Tassev, M. Zaldarriaga, and D. J. Eisenstein, Solving large scale structure in ten easy steps with COLA, J. Cosmol. Astropart. Phys. 06 (2013) 036.

[4] A. Izard, M. Crocce, and P. Fosalba, ICE-COLA: Towards fast and accurate synthetic galaxy catalogues optimizing a quasi-N-body method, Mon. Not. R. Astron. Soc. 459, 2327 (2016).

[5] F. Leclercq, B. Faure, G. Lavaux, B. D. Wand elt, A. H. Jaffe, A. F. Heavens, W. J. Percival, and C. Noûs, Perfectly parallel cosmological simulations using spatial comoving Lagrangian acceleration, Astron. Astrophys. 639, A91 (2020).

[6] Y. Feng, M.-Y. Chu, U. Seljak, and P. McDonald, FASTPM: A new scheme for fast simulations of dark matter and haloes, Mon. Not. R. Astron. Soc. 463, 2273 (2016).

[7] P. Monaco, E. Sefusatti, S. Borgani, M. Crocce, P. Fosalba, R. K. Sheth, and T. Theuns, An accurate tool for the fast generation of dark matter halo catalogues, Mon. Not. R. Astron. Soc. 433, 2389 (2013).

[8] M. Manera et al., The clustering of galaxies in the SDSS-III Baryon oscillation spectroscopic survey: A large sample of mock galaxy catalogues, Mon. Not. R. Astron. Soc. 428, 1036 (2013).

[9] F. S. Kitaura, G. Yepes, and F. Prada, Modelling baryon acoustic oscillations with perturbation theory and stochastic halo biasing., Mon. Not. R. Astron. Soc. 439, L21 (2014).

[10] S. Avila, S. G. Murray, A. Knebe, C. Power, A. S. G. Robotham, and J. Garcia-Bellido, HALOGEN: A tool for fast generation of mock halo catalogues, Mon. Not. R. Astron. Soc. 450, 1856 (2015).
[11] G. Stein, M. A. Alvarez, and J. R. Bond, The mass-peak patch algorithm for fast generation of deep all-sky dark matter halo catalogues and its N-body validation, Mon. Not. R. Astron. Soc. 483, 2236 (2019).

[12] G. Stein, georgestein/ml-in-cosmology: Machine learning in cosmology (2020), https://doi.org/10.5281/zenodo .4024768 .

[13] P. Berger and G. Stein, A volumetric deep convolutional neural network for simulation of mock dark matter halo catalogues, Mon. Not. R. Astron. Soc. 482, 2861 (2019).

[14] S. He, Y. Li, Y. Feng, S. Ho, S. Ravanbakhsh, W. Chen, and B. Póczos, Learning to predict the cosmological structure formation, Proc. Natl. Acad. Sci. U.S.A. 116, 13825 (2019).

[15] M. Bernardini, L. Mayer, D. Reed, and R. Feldmann, Predicting dark matter halo formation in N-body simulations with deep regression networks, Mon. Not. R. Astron. Soc. 496, 5116 (2020).

[16] C. Modi, Y. Feng, and U. Seljak, Cosmological reconstruction from galaxy light: Neural network based lightmatter connection, J. Cosmol. Astropart. Phys. 10 (2018) 028.

[17] E. Giusarma, M. Reyes Hurtado, F. Villaescusa-Navarro, S. He, S. Ho, and C. Hahn, Learning neutrino effects in cosmology with convolutional neural networks, arXiv: 1910.04255 .

[18] F. List, I. Bhat, and G. F. Lewis, A black box for dark sector physics: predicting dark matter annihilation feedback with conditional GANs, Mon. Not. R. Astron. Soc. 490, 3134 (2019).

[19] D. Kodi Ramanah, T. Charnock, and G. Lavaux, Painting halos from cosmic density fields of dark matter with physically motivated neural networks, Phys. Rev. D 100, 043515 (2019).

[20] T. Tröster, C. Ferguson, J. Harnois-Déraps, and I. G. McCarthy, Painting with baryons: Augmenting N-body simulations with gas using deep generative models, Mon. Not. R. Astron. Soc. 487, L24 (2019). 
[21] X. Zhang, Y. Wang, W. Zhang, Y. Sun, S. He, G. Contardo, F. Villaescusa-Navarro, and S. Ho, From dark matter to galaxies with convolutional networks, arXiv:1902.05965.

[22] I. J. Goodfellow, J. Pouget-Abadie, M. Mirza, B. Xu, D. Warde-Farley, S. Ozair, A. Courville, and Y. Bengio, Generative adversarial networks, arXiv:1406.2661.

[23] M. Mustafa, D. Bard, W. Bhimji, Z. Lukić, R. Al-Rfou, and J. M. Kratochvil, CosmoGAN: Creating high-fidelity weak lensing convergence maps using generative adversarial networks, Comput. Astrophys. Cosmol. 6, 1 (2019).

[24] A. C. Rodríguez, T. Kacprzak, A. Lucchi, A. Amara, R. Sgier, J. Fluri, T. Hofmann, and A. Réfrégier, Fast cosmic web simulations with generative adversarial networks, Comput. Astrophys. Cosmol. 5, 4 (2018).

[25] J. Zamudio-Fernandez, A. Okan, F. Villaescusa-Navarro, S. Bilaloglu, A. Derin Cengiz, S. He, L. Perreault Levasseur, and S. Ho, HIGAN: Cosmic neutral hydrogen with generative adversarial networks, arXiv:1904.12846.

[26] D. Kodi Ramanah, T. Charnock, F. Villaescusa-Navarro, and B. D. Wandelt, Super-resolution emulator of cosmological simulations using deep physical models, Mon. Not. R. Astron. Soc. 495, 4227 (2020).

[27] N. Perraudin, A. Srivastava, A. Lucchi, T. Kacprzak, T. Hofmann, and A. Réfrégier, Cosmological N-body simulations: A challenge for scalable generative models, Comput. Astrophys. Cosmol. 6, 5 (2019).

[28] D. L. Ruderman, The statistics of natural images, Network Comput. Neural Syst. 5, 517 (1994).

[29] M. Mirza and S. Osindero, Conditional generative adversarial nets, arXiv:1411.1784.

[30] http://yann.lecun.com/exdb/mnist/.

[31] T. Karras, S. Laine, and T. Aila, A style-based generator architecture for generative adversarial networks, arXiv:1812 .04948 .

[32] A. Brock, J. Donahue, and K. Simonyan, Large scale GAN training for high fidelity natural image synthesis, arXiv: 1809.11096.

[33] J. R. Bond, L. Kofman, and D. Pogosyan, How filaments of galaxies are woven into the cosmic web, Nature (London) 380, 603 (1996).

[34] https://wwwmpa.mpa-garching.mpg.de/gadget/.

[35] A. G. Sánchez et al., The clustering of galaxies in the SDSSIII Baryon oscillation spectroscopic survey: Cosmological constraints from the full shape of the clustering wedges, Mon. Not. R. Astron. Soc. 433, 1202 (2013).

[36] J. Wu, C. Zhang, T. Xue, W. T. Freeman, and J. B. Tenenbaum, Learning a probabilistic latent space of object shapes via 3D generative-adversarial modeling, arXiv:1610 .07584 .

[37] A. Radford, L. Metz, and S. Chintala, Unsupervised representation learning with deep convolutional generative adversarial networks, arXiv:1511.06434.

[38] R. Singh, P. Turaga, S. Jayasuriya, R. Garg, and M. W. Braun, Non-parametric priors for generative adversarial networks, arXiv:1905.07061.
[39] D. P. Kingma and J. Ba, Adam: A method for stochastic optimization, arXiv:1412.6980.

[40] M. Arjovsky, S. Chintala, and L. Bottou, Wasserstein GAN, arXiv:1701.07875.

[41] I. Gulrajani, F. Ahmed, M. Arjovsky, V. Dumoulin, and A. Courville, Improved training of wasserstein GANs, arXiv: 1704.00028.

[42] https://pytorch.org/.

[43] https://github.com/RichardFeder/gan-work.

[44] B. Pandey and S. Sarkar, Testing homogeneity in the sloan digital sky survey data release twelve with shannon entropy, Mon. Not. R. Astron. Soc. 454, 2647 (2015).

[45] R. S. Gonçalves, G. C. Carvalho, J. Bengaly, C. A. P. Bengaly, J. C. Carvalho, A. Bernui, J. S. Alcaniz, and R. Maartens, Cosmic homogeneity: A spectroscopic and model-independent measurement, Mon. Not. R. Astron. Soc. 475, L20 (2018).

[46] N. Hand, Y. Feng, F. Beutler, Y. Li, C. Modi, U. Seljak, and Z. Slepian, nbodykit: Massively parallel, large-scale structure toolkit (2019), ascl:1904.027.

[47] M. Bartelmann, The dark Universe, Rev. Mod. Phys. 82, 331 (2010).

[48] https://github.com/franciscovillaescusa/Pylians.

[49] P. Betzler and S. Krippendorf, Connecting dualities and machine learning, Fortschr. Phys. 68, 2000022 (2020).

[50] T. Schlegl, P. Seeböck, S. M. Waldstein, U. SchmidtErfurth, and G. Langs, Unsupervised anomaly detection with generative adversarial networks to guide marker discovery, arXiv:1703.05921.

[51] V. Böhm, F. Lanusse, and U. Seljak, Uncertainty quantification with generative models, arXiv:1910.10046.

[52] C. Esteves, C. Allen-Blanchette, A. Makadia, and K. Daniilidis, Learning SO(3) equivariant representations with spherical CNNs, arXiv:1711.06721.

[53] T. S. Cohen, M. Weiler, B. Kicanaoglu, and M. Welling, Gauge equivariant convolutional networks and the icosahedral CNN, arXiv:1902.04615.

[54] L. Falorsi, P. de Haan, T. R. Davidson, N. De Cao, M. Weiler, P. Forré, and T. S. Cohen, Explorations in homeomorphic variational auto-encoding, arXiv:1807.04689.

[55] http://www.tacc.utexas.edu.

[56] C. R. Harris et al., Array programming with NumPy, Nature (London) 585, 357 (2020).

[57] S. G. Murray, powerbox: A Python package for creating structured fields with isotropic power spectra, J. Open Source Software 3, 850 (2018).

[58] M. Heusel, H. Ramsauer, T. Unterthiner, B. Nessler, and S. Hochreiter, GANs trained by a two time-scale update rule converge to a local nash equilibrium, arXiv:1706.08500.

[59] T. Salimans, I. Goodfellow, W. Zaremba, V. Cheung, A. Radford, and $\mathrm{X}$. Chen, Improved techniques for training GANs, arXiv:1606.03498.

[60] S. Azadi, C. Olsson, T. Darrell, I. Goodfellow, and A. Odena, Discriminator rejection sampling, arXiv:1810 .06758 . 